National Burøau or Ltandaras

Library, N. ?. Btag

MAR 231964

NBS

Eechnical Note

n. 210

\title{
AN ATLAS OF SOLAR FLARE EFFECTS OBSERVED ON LONG VLF PATHS DURING 1961
}

C. J. CHILTON, F. K. STEELE, AND D. D. CROMBIE

U. S. DEPARTMENT OF COMMERCE NATIONAL BUREAU OF STANDARDS 


\section{,}




\title{
NATIONAL BUREAU OF STANDARDS Eechnical Mote 210 Issued March 13, 1964
}

\section{AN ATLAS OF SOLAR FLARE EFFECTS OBSERVED ON LONG VLF PATHS DURING 1961}

\author{
C. J. Chilton, F. K. Steele, and D. D. Crombie \\ Central Radio Propagation Laboratory \\ National Bureau of Standards \\ Boulder, Colorado
}

NBS Technical Notes are designed to supplement the Bureau's regular publications program. They provide a means for making available scientific data that are of transient or limited interest. Technical Notes may be listed or referred to in the open literature.

For sale by the Superintendent of Documents, U. S. Government Printing Office Washington, D.C. 20402

Price $30 \notin$ 

Abstract ...................... . . 1

1. Introduction . . . . . . . . . . . . . . . 1

2. Experimental Observations and Description of the Data . . 2

3. Acknowledgments ................. . . 4

4. References .................... 4

Figures . . . . . . . . . . . . . . . . . 5

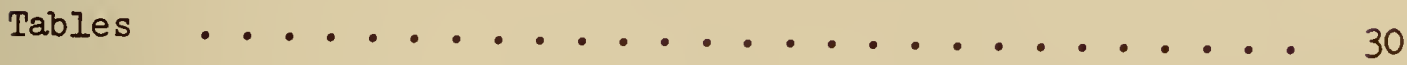



AN ATLAS OF SOLAR FLARE EFFECTS OBSERVED ON LONG VLF PATHS DURING 1961

C. J. Chilton, F. K. Steele, and D. D. Crombie

Effects produced by 37 solar flares

on four long VLF paths during 1961 are shown and tabulated.

\section{Introduction}

Since early in 1961, the phase and amplitude of various very-lowfrequency transmissions have been monitored by the Boulder Laboratories of the National Bureau of Standards; the NBS field site at Maui, Hawaii; the Battelle Research Institute, Frankfurt, Germany; and the Geophysical Institute, College, Alaska. Observations of three phasestabilized VLF transmissions over four long propagation, paths were made at the above receiving sites using the VLF signals radiated from NBA $(18 \mathrm{kc} / \mathrm{s})$, Balboa, Panama; NPG $(18.6 \mathrm{kc} / \mathrm{s})$, Seattle, Washington; and $\operatorname{GBR}(16 \mathrm{kc} / \mathrm{s})$, Rugby, England. The propagation paths, their respective lengths and geographic orientations are shown in figure 1. This VLF transmission network samples the variations occurring over approximately one quarter of the earth's surface and thus provides an excellent means for studying the effects of solar flare produced ionization over a large area of the ionosphere [Chilton, et al., 1963], as well as the normal day-to-night variation in ionospheric height.

Of the known perturbations which are observed in the recorded phase of a VLF transmission, the most easily recognizable are the Sudden Phase Anomalies (SPA's) [Bracewell and Straker, 1949], which are known to be produced by lonjzing radiations emitted from the sun's chromosphere. These chromospheric flares, usually referred to as solar flares, are short-lived sudden increases in light intensity generally observed near sunspots. Optical observations show that almost all flares follow the same pattern, a rapid rise to peak intensity followed by a short period of peak intensity and a slow return to the preflare conditions. Typical flares have an onset time that varies from $I$ to 30 minutes and the return to normal requires about 30 minutes to 2 or 3 hours. In order to provide a measure of their relative magnitude, flares have been divided into classes of importance ( $1,2,3$ and $3+$ ) according to their area and brightness. The surface area of Class 1 flares is on the order of $10^{-4}$ of the solar hemisphere, corresponding to a diameter of about $10^{9} \mathrm{~cm}$. The brightness factor is obtained by photometrically observing the $\mathrm{Ha}$ line of the solar spectrum. The magnitude of the associated phase advance is apparently closely related to the increase in solar radiation, its energy spectrum, its angle of incidence at the lower regions of the ionosphere, and to the length of path over which a lowering of the apparent height of reflection occurs. 
It is the sole purpose of this note to provide examples of the solar flare effects observed on the paths listed above during 1961. Emphasis has been placed on those flares for which observations are available on more than one path.

\section{Experimental Observations and Description of the Data}

The propagation paths are shown in figure 1. All of the paths except one (NPG-College) are sufficiently long to make it reasonable to assume that only one waveguide mode [Wait, 1962] is present. The data obtained with signals propagating along these paths show that during a solar flare the signals received over the sunlit paths exhibit sudden phase anomalies, which are not identical, either in magnitude or duration. These observations are illustrated in figures 2 through 25. These illustrations are photographic copies of the original records obtained between May 1 and December 2, 1961. In each case the phase and amplitude traces and the directions of phase advance and amplitude increase are identified. The records shown were chosen after examining all of the recorded data. Subsequently optical observations of solar flares as listed in the CRPL Series F, Part B (SolarGeophysical Data) Bulletins were examined and times of optical sightings were obtained and added to the figures. In addition, the SolarGeophysical Data were examined independently and times of all Class 1 or greater flares were obtained. The VLF data were then re-examined for solar flare effects at these times. Flares for which observations on two or more paths were available are included in figures 2-25, although some other flares are included because of their proximity in time to flares for which there are two or more observations. These figures contain effects observed during 37 flares. Included in the figures are some records for which there was no visual sighting; these records are very similar to those which are associated with flares and have been included for this reason. Also included are several records for which all the paths are not totally sunlit, but which nevertheless show an appreciable effect.

The SPA's and optical classification of the associated solar flares have been listed in chronological order in table 1, which in addition contains some of the more important characteristics of the SPA's, as scaled from the figures. Following the date, the optical class and time of optical sighting are listed. The next three golumns list the time of first appearance of an effect on the phase records, the time of maximum effect, and the time of return to normal. Then the size of the phase anomaly $\Delta \phi$ in degrees, and in microseconds $(\Delta t)$ is listed. The latter quantity, $\Delta t$ is obtained from the relation

$$
\Delta t=\frac{\Delta \phi}{0.36} \cdot \frac{1}{f}
$$


where $f$ is in $\mathrm{kc} / \mathrm{s}$. The size of the phase change $\Delta \phi$ produced by a given depression $\Delta h$ of the ionosphere is not particularly useful in itself since it depends on the path length and the frequency of the signal, as well as on $\Delta h$. Thus $\Delta h$ is a better index of the magnitude of the solar flare effect. So in the next column, values of $\Delta h$ calculated in the manner described by Wait [1959, 1961] are given for all paths except NPG - College which, as noted above, is too short to assume that only one waveguide mode is present. These values of $\Delta h$ are obtained on the assumption that the flare produces a constant depression along the whole path. This of course is hardly likely, since it would be reasonable to assume that the flare would produce the greatest effects at the sub-solar point, the effects becoming smaller as the zenith angle of sun increases. This might be corrected by relating the zenith angle $(X)$ to the calculated value of $\Delta h$ since it has been shown [Chilton, et al., 1963] that when the observed $\Delta h$ obtained on the above basis is plotted semilogarithmically against 1/ $\overline{\operatorname{Cos} x}$, (where $\overline{\cos x}$ is the average value of $\cos x$ along the propagation path), a straight line results. Thus the table contains values of average $X$ and average $\cos x$ which are listed in the last two columns.

Table 1 also contains estimates of the maximum change of $\phi$ and ( $d \phi / d t)$ observed on each path, for each flare. Observed values of $\mathrm{d} \phi / \mathrm{dt}$ vary from as much as $90^{\circ} / \mathrm{min}$ to as little as $1^{\circ} / \mathrm{min}$.

In view of the wide geographical distribution of the paths on which these observations have been made, and of the distribution in time of occurrences of the flares, it seems reasonable to regard these observations as a sample of typical flare observations which might be made on any path at any time. Therefore, the scaled data from table 1 have been separated according to the optical classification of the originating flare. Table 2 thus contains for each flare of Class 1,2 or 3, the maximum, mean, and minimum phase shifts, together with the phase shifts exceeded by $25 \%, 50 \%$, and $75 \%$ of the observations. Similarly, table 3 contains corresponding values of the rate of change of phase. It can be seen from these tables that the mean and upper quartile of both the phase change and maximum rate of change of phase increase with increasing optical classification. On the other hand, this tendency is not shown clearly by either the maximum phase change or the maximum rate of change of phase. This is possibly because the change in height of the D region is related to the solar zenith angle [Chilton, et al., 1963]. 


\section{Acknowledgments}

The observations at College were made under the supervision of Dr. H. F. Bates at the Geophysical Institute, University of Alaska. Those at Frankfurt were made under the supervision of $\mathrm{Dr}$. J. Eitzenberger of the Battelle Institute. The observations at Maui were made by Sada Katahara, while those at Boulder were under the supervision of A. H. Diede. The VIF program at Frankfurt, College, and Boulder is supported by the Advanced Research Projects Agency, Washington, D. C.

\section{References}

Bracewell, R. N., and T. W. Straker (1949), The study of solar flares by means of very long radio waves, Monthly Notices, Roy. Ast. Soc. $109,28$.

Chilton, C. J., F. K. Steele, and R. B. Norton (1963), VLF phase observations of solar flare ionization in the $D$ region of the ionosphere, J. Geophys. Res. 68, 5421-5435.

Wait, J. R. (Nov. 5, 1959), Diurnal change of ionospheric heights deduced from phase velocity measurements at VIF, Proc. IRE 47, 998.

Wait, J. R. (1962), Comments on a paper by W. D. Westfall, Prediction of VIF diurnal phase changes and solar flare effect, J. Geophys. Res. 67, 916. 


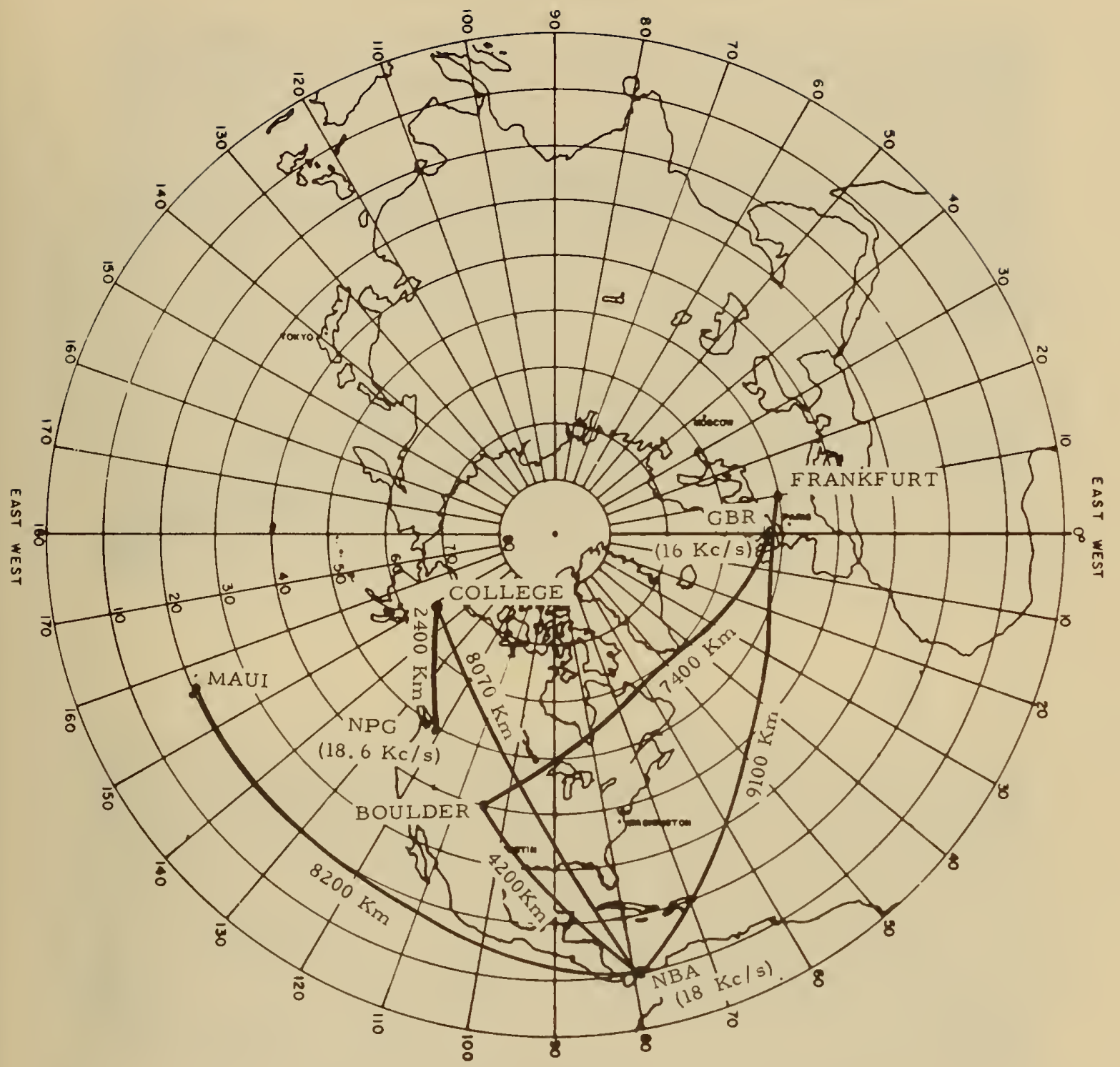

Figure 1. Map showing the paths and transmitter frequencies used in this Note. 


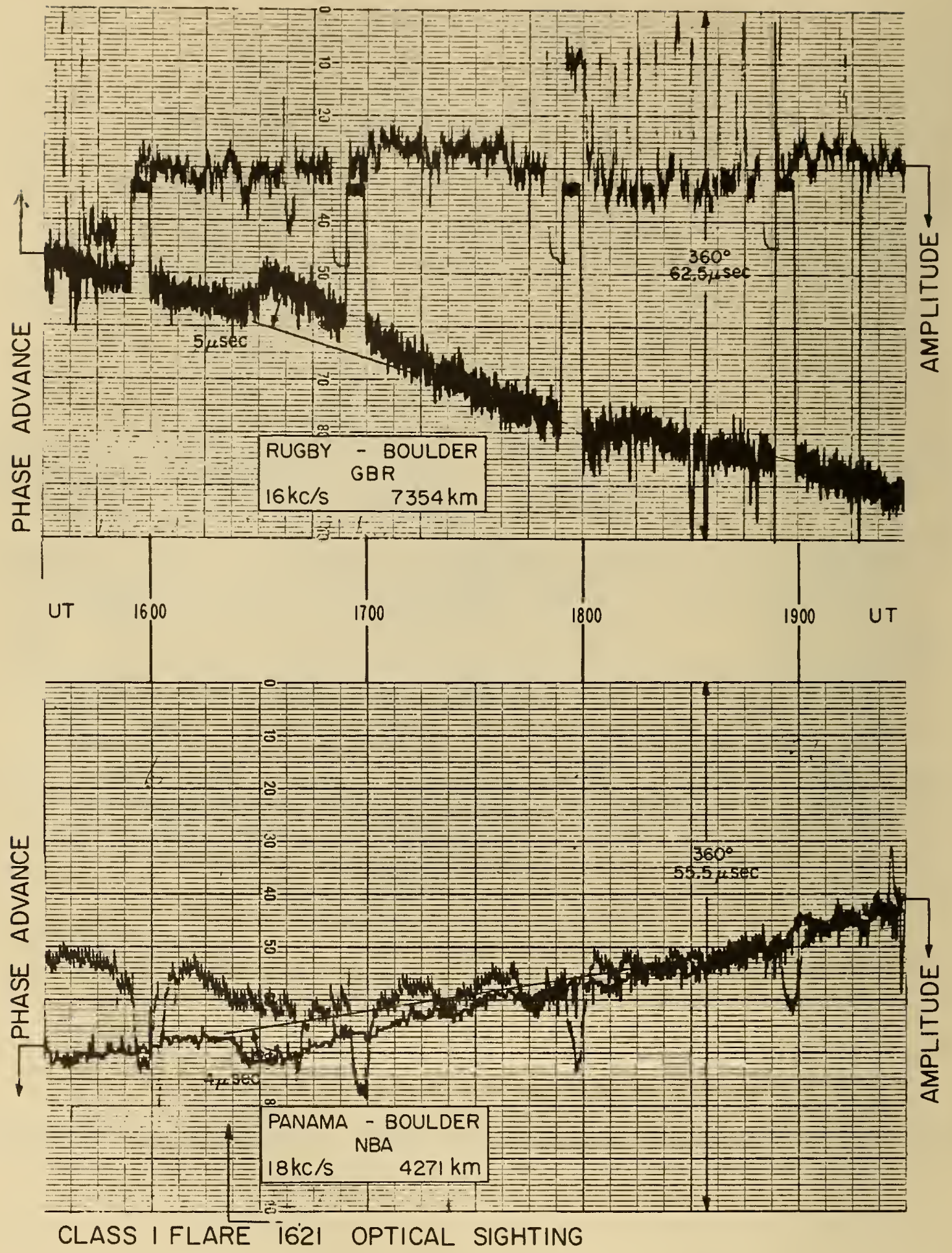

Figure 2 
SUDDEN PHASE ANOMALY 5 -JUNE $196 \mid$ UT

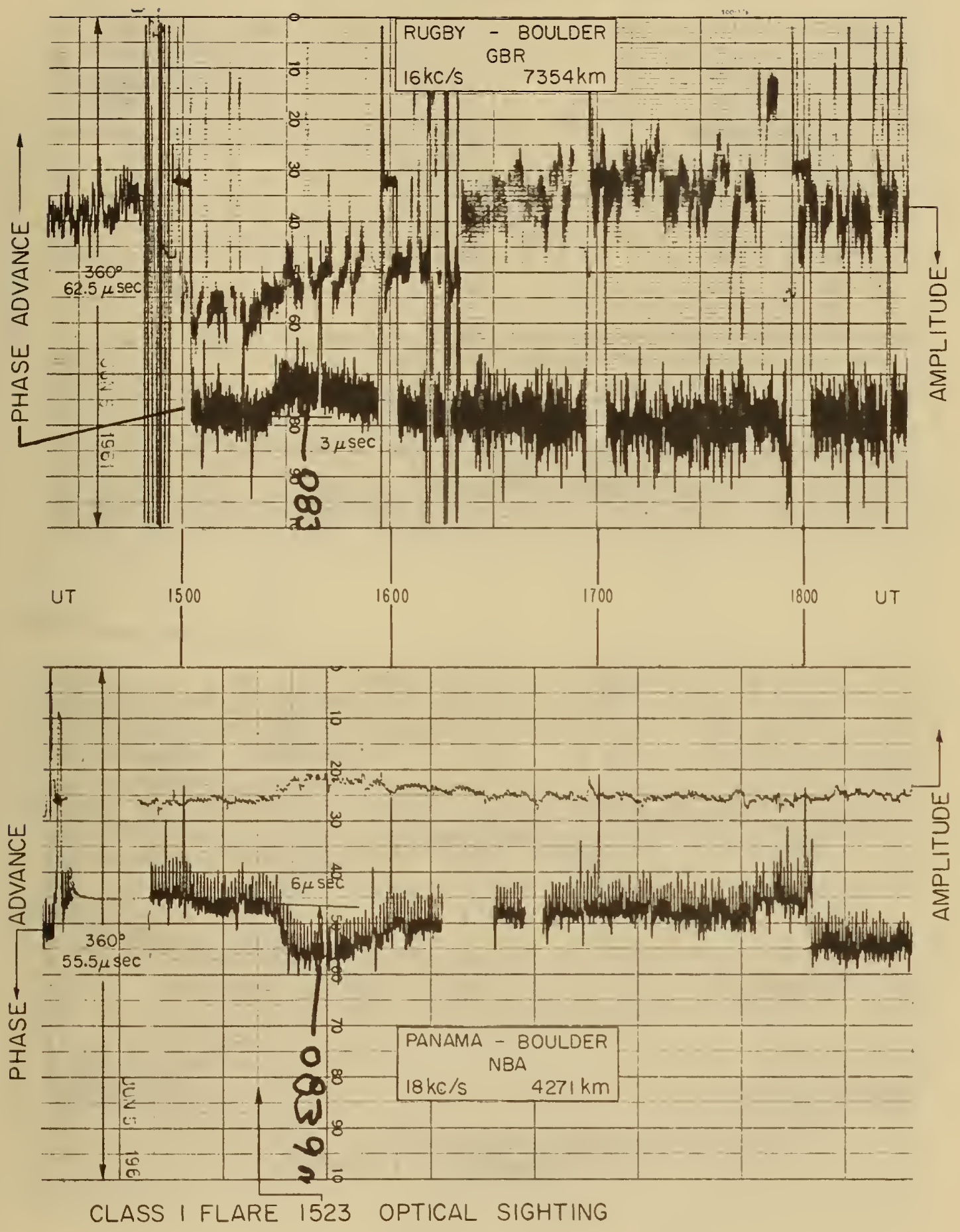

Figure 3 
SUDDEN PHASE ANOMALY ||-JULY I96| UT

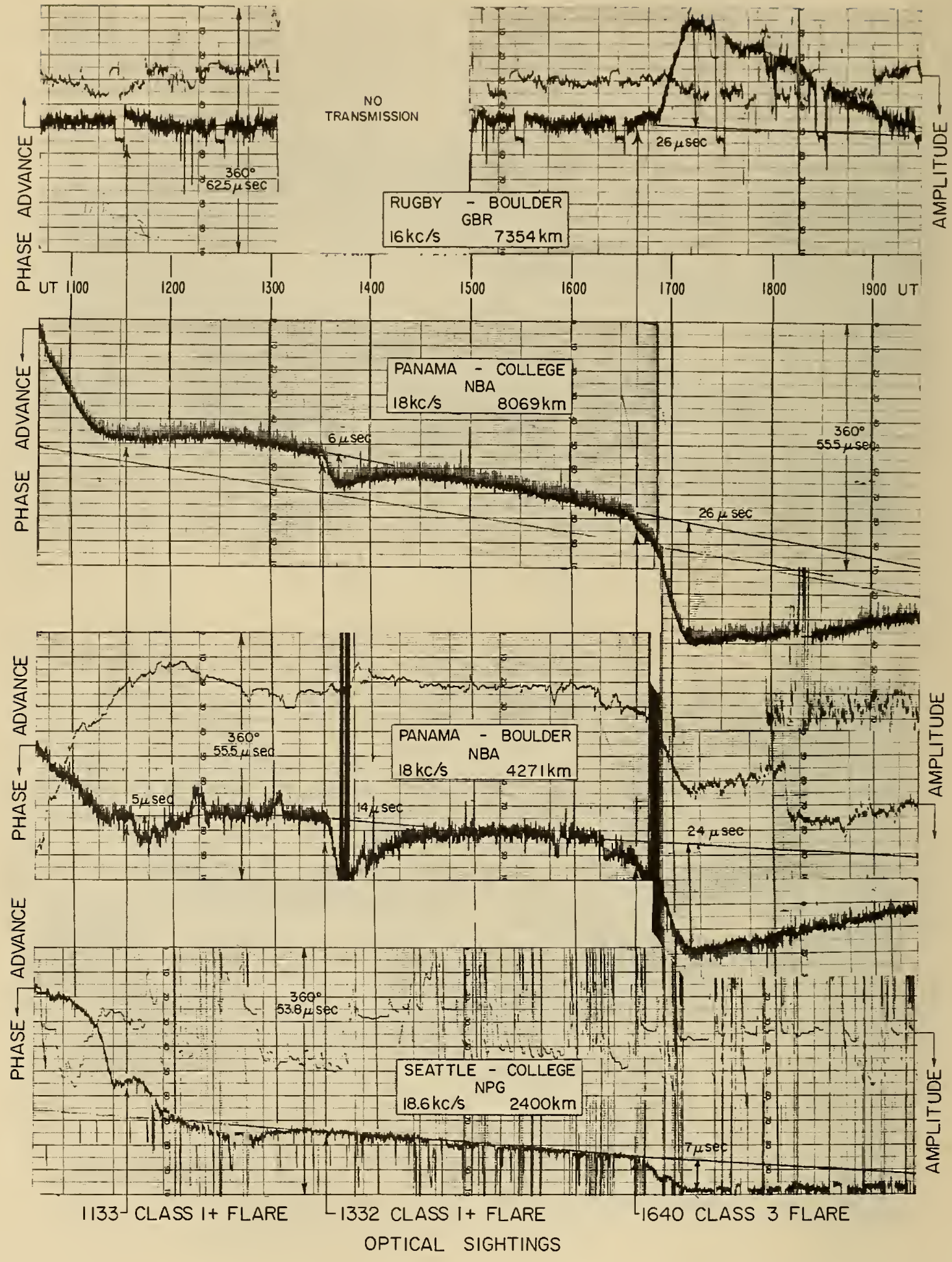

Figure 4 

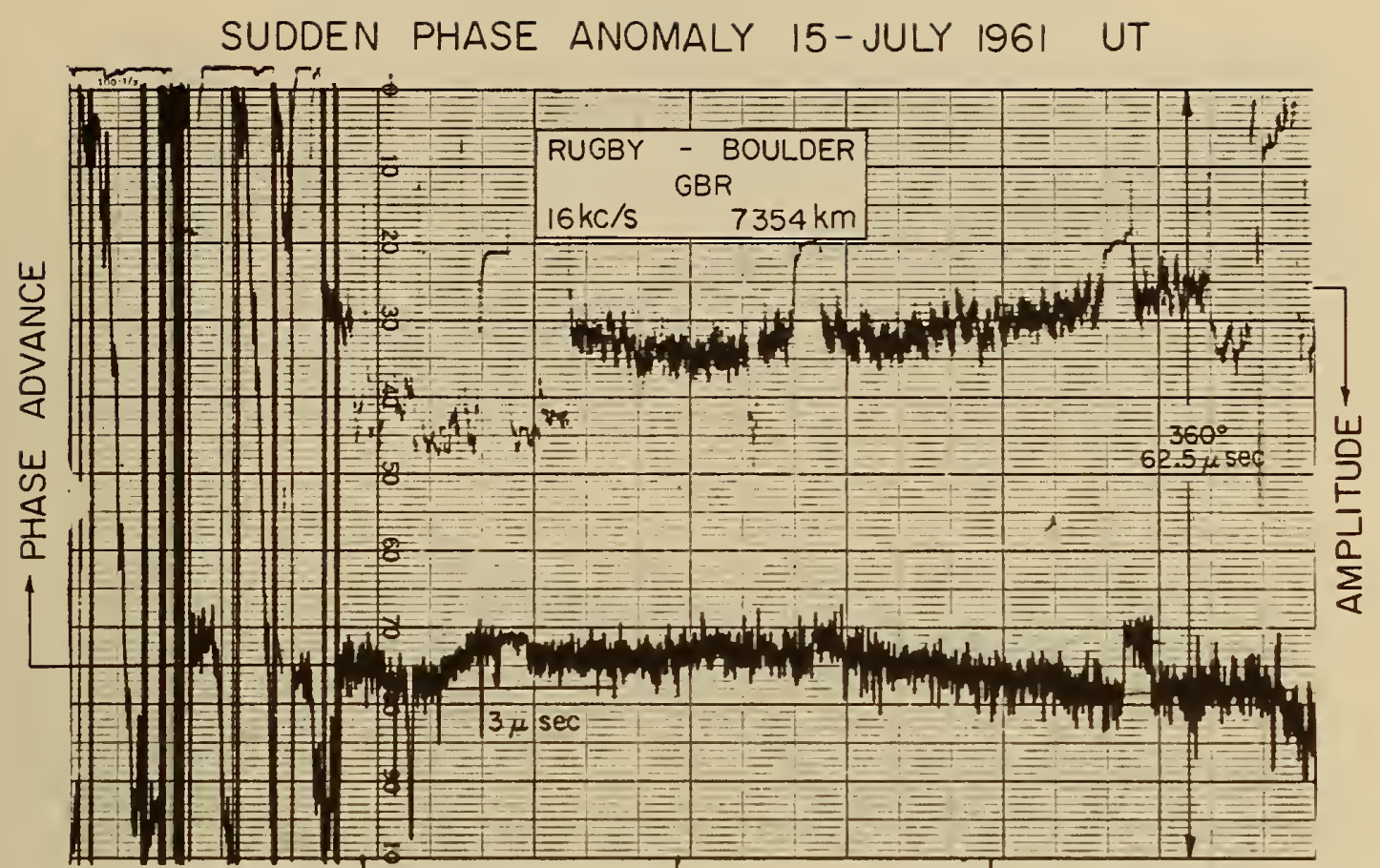

UT

1500

1600

1700

UT

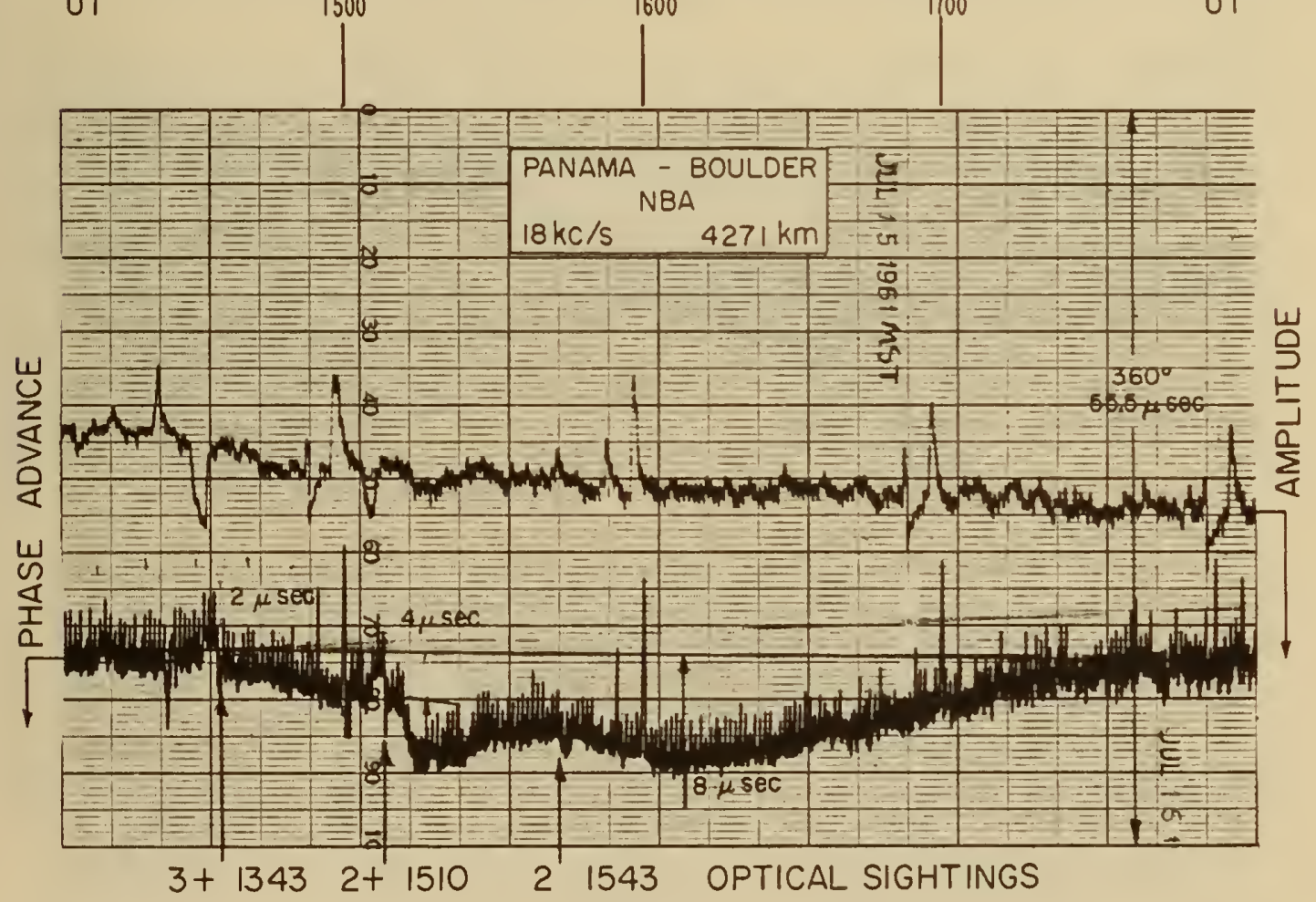

Figure 5 

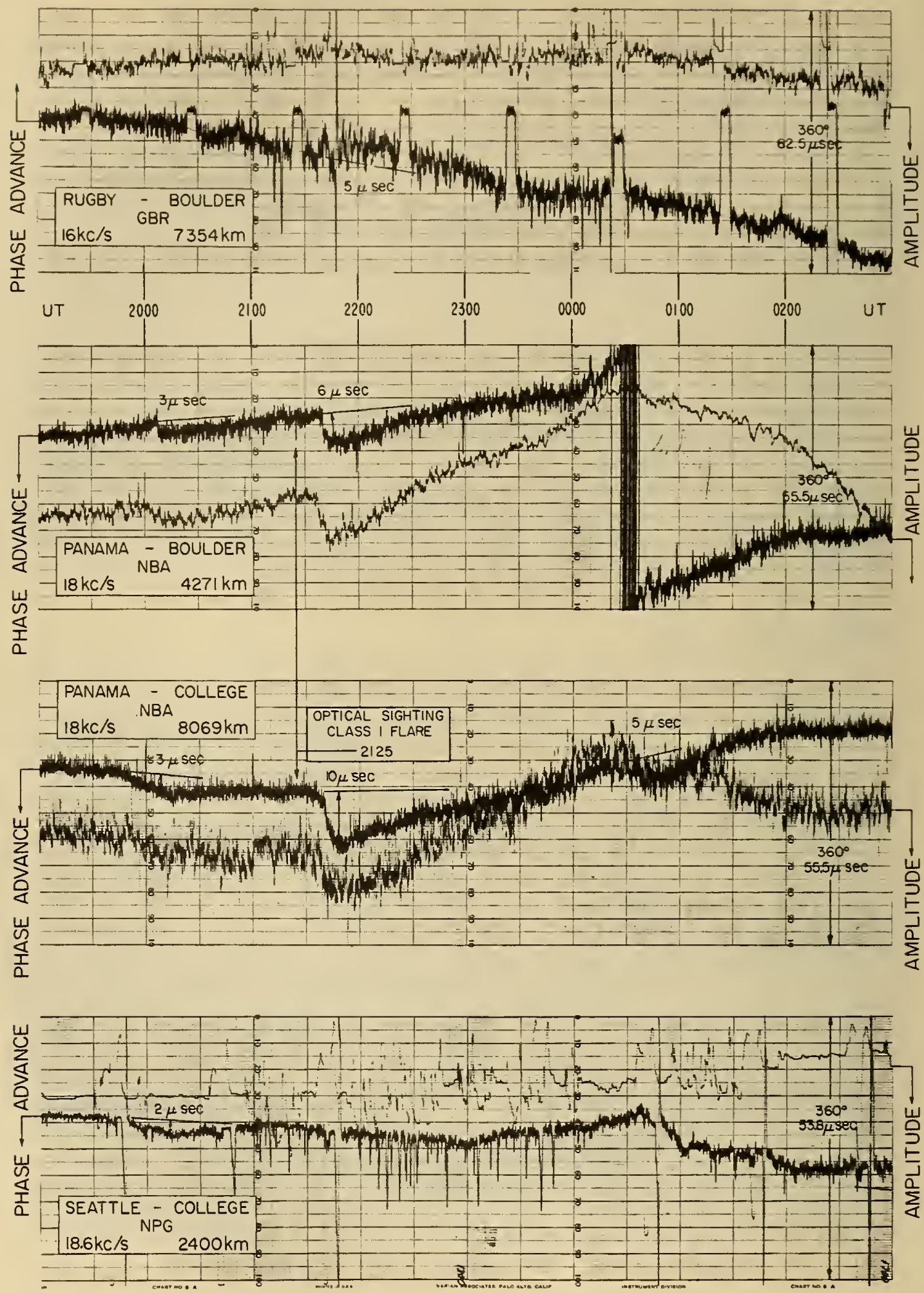

Figure 6 


\section{SUDDEN PHASE ANOMALY 18-JULY 1961 UT}
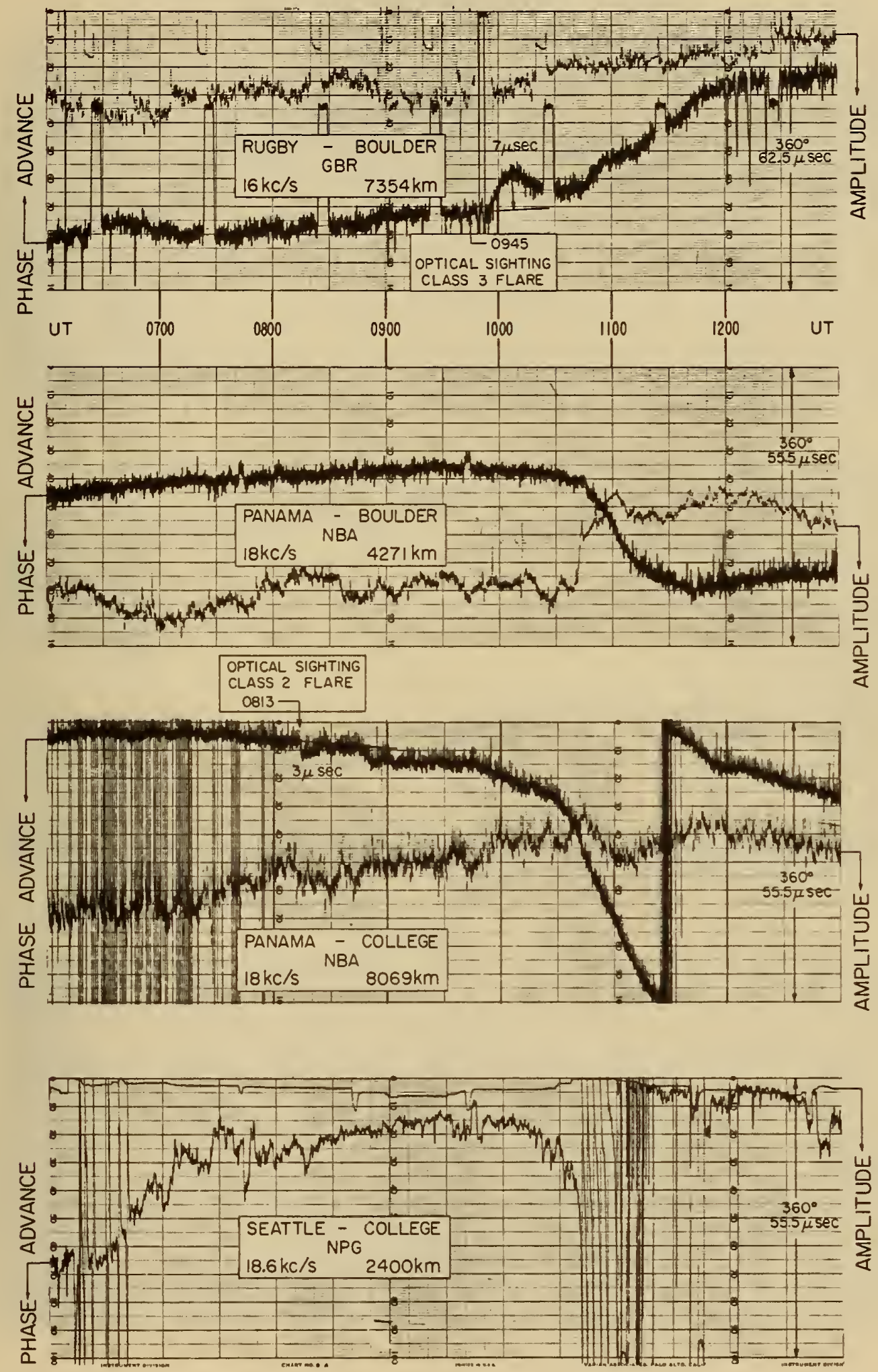

Figure 7 
SUDDEN PHASE ANOMALY 20-JULY 1961 UT
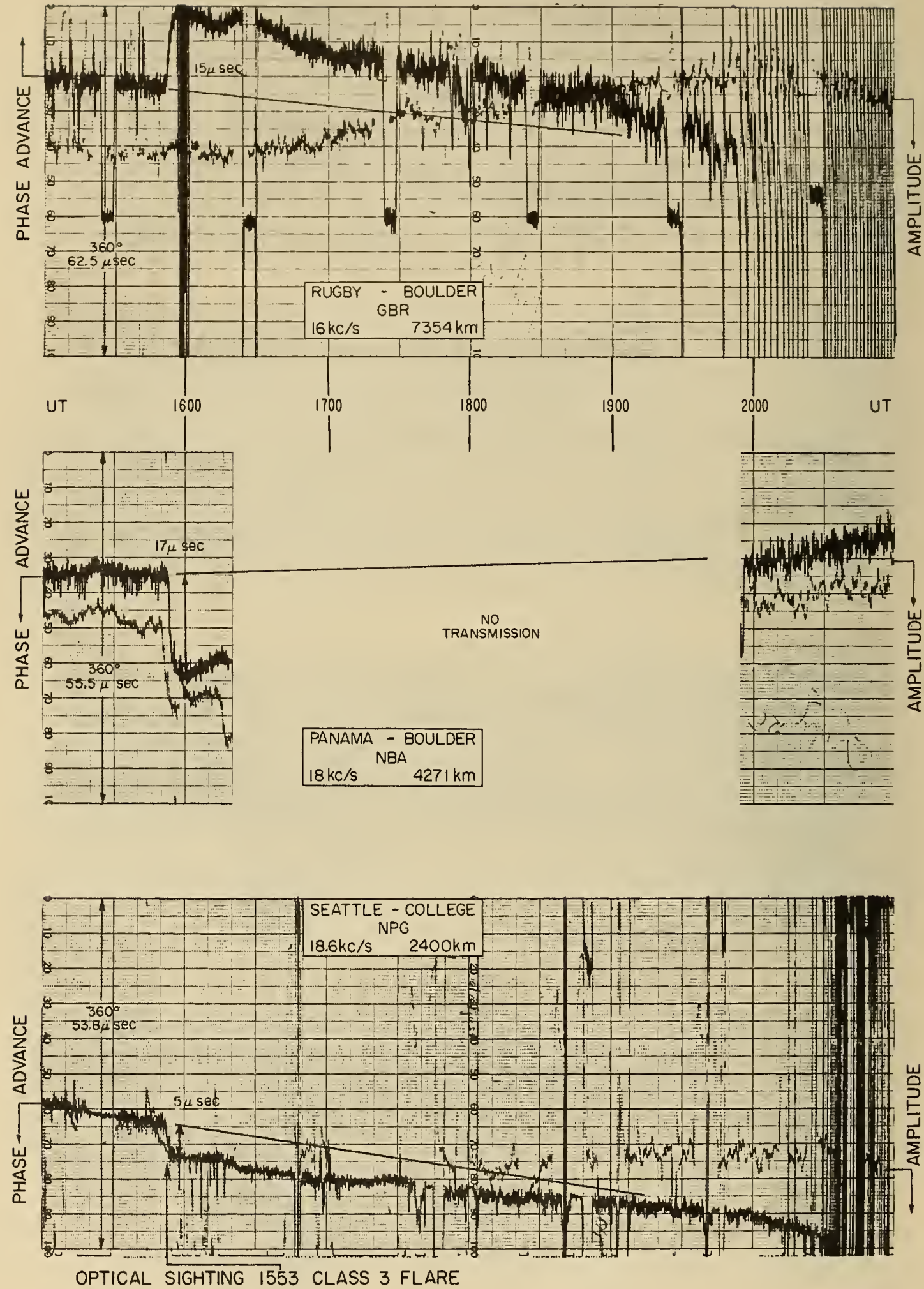

Figure 8 
SUDDEN PHASE ANOMALY 21-JULY 1961 UT
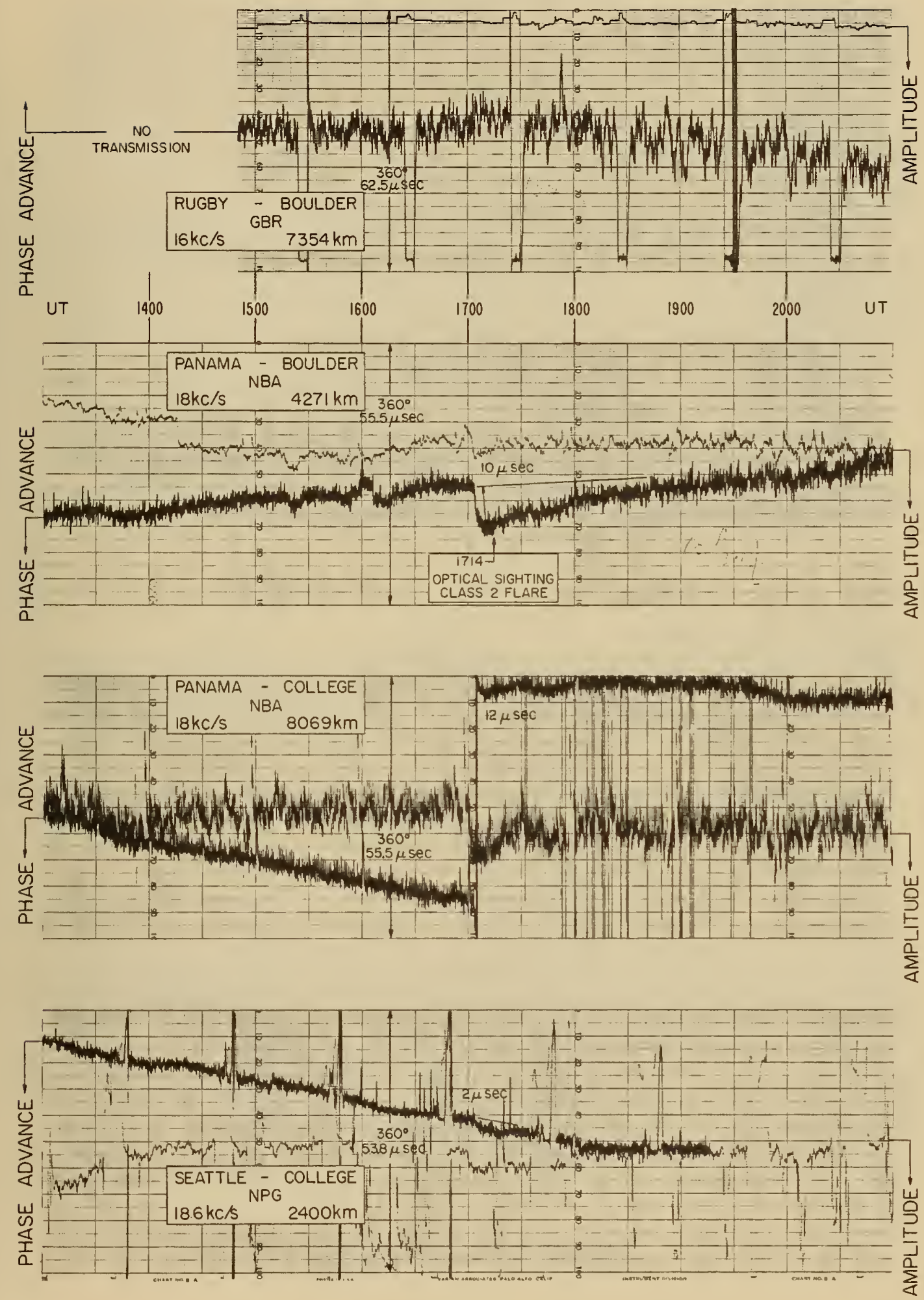

Figure 9 
SUDDEN PHASE ANOMALY 23-JULY 1961 UT

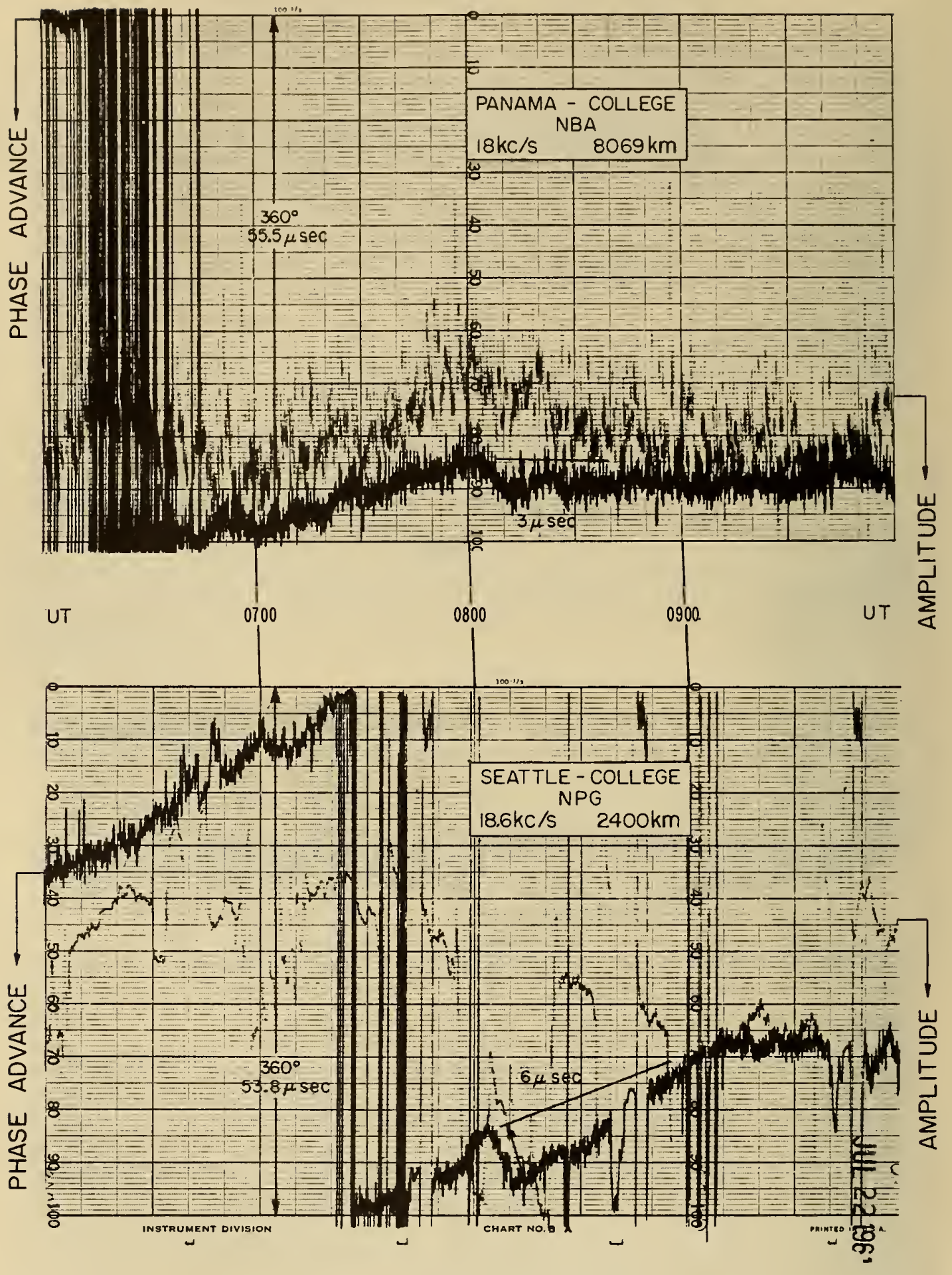

NO OPTICAL SIGHTING

Figure 10 

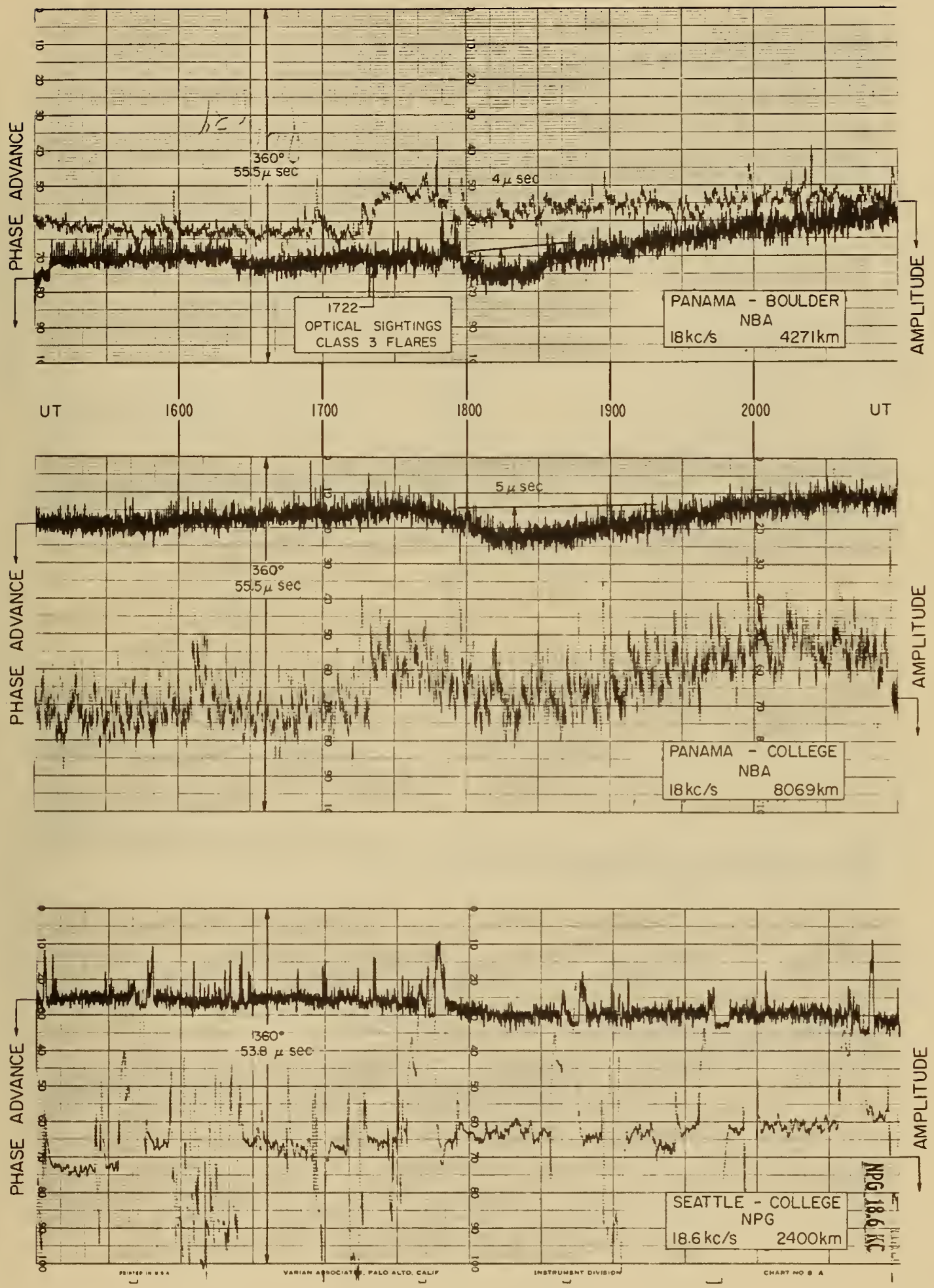

Figure 11 
SUDDEN PHASE ANOMALY 15-AUGUST 1961 UT

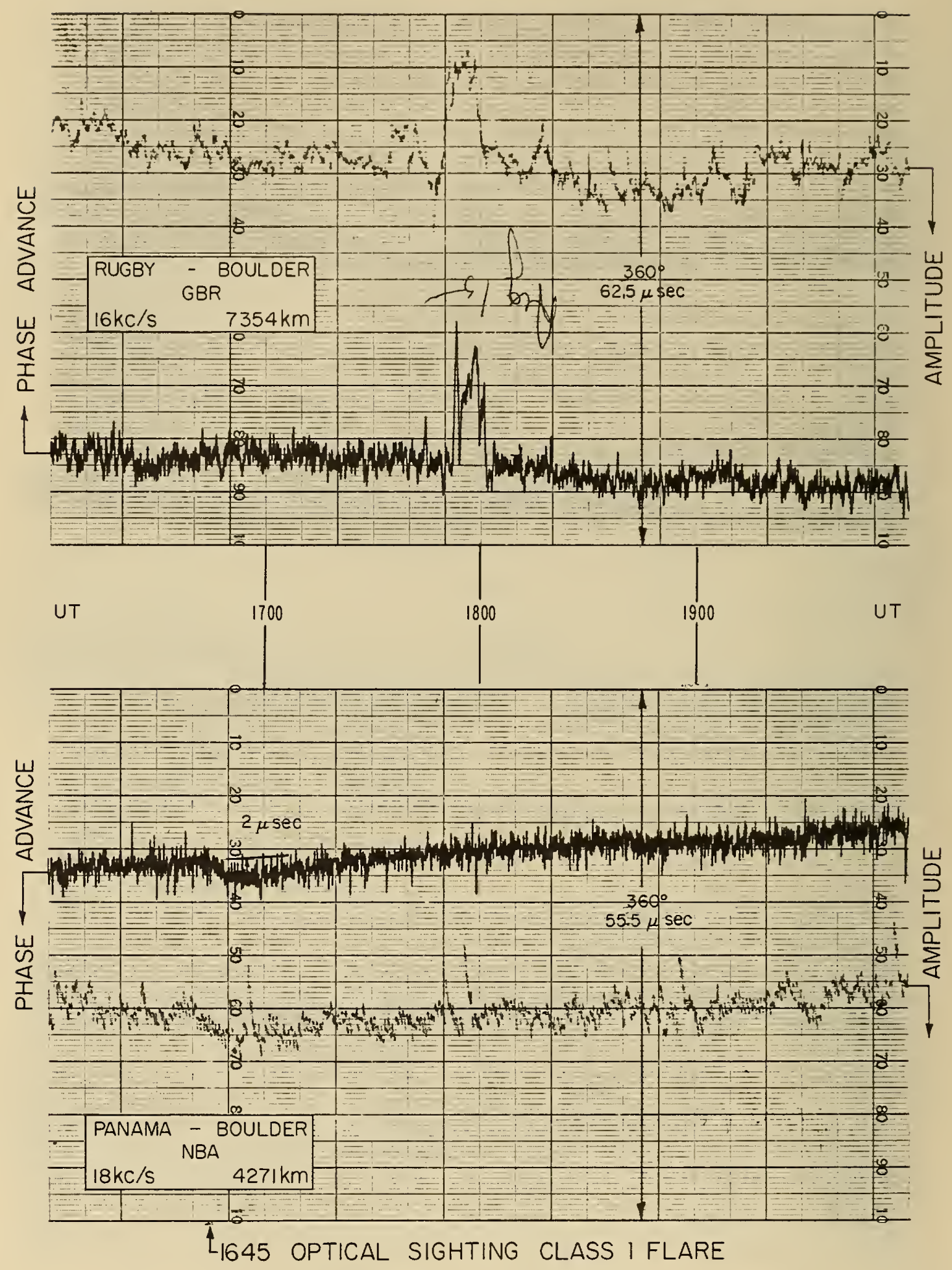

Figure 12 
SUDDEN PHASE ANOMALY 18-AUGUST 1961 UT

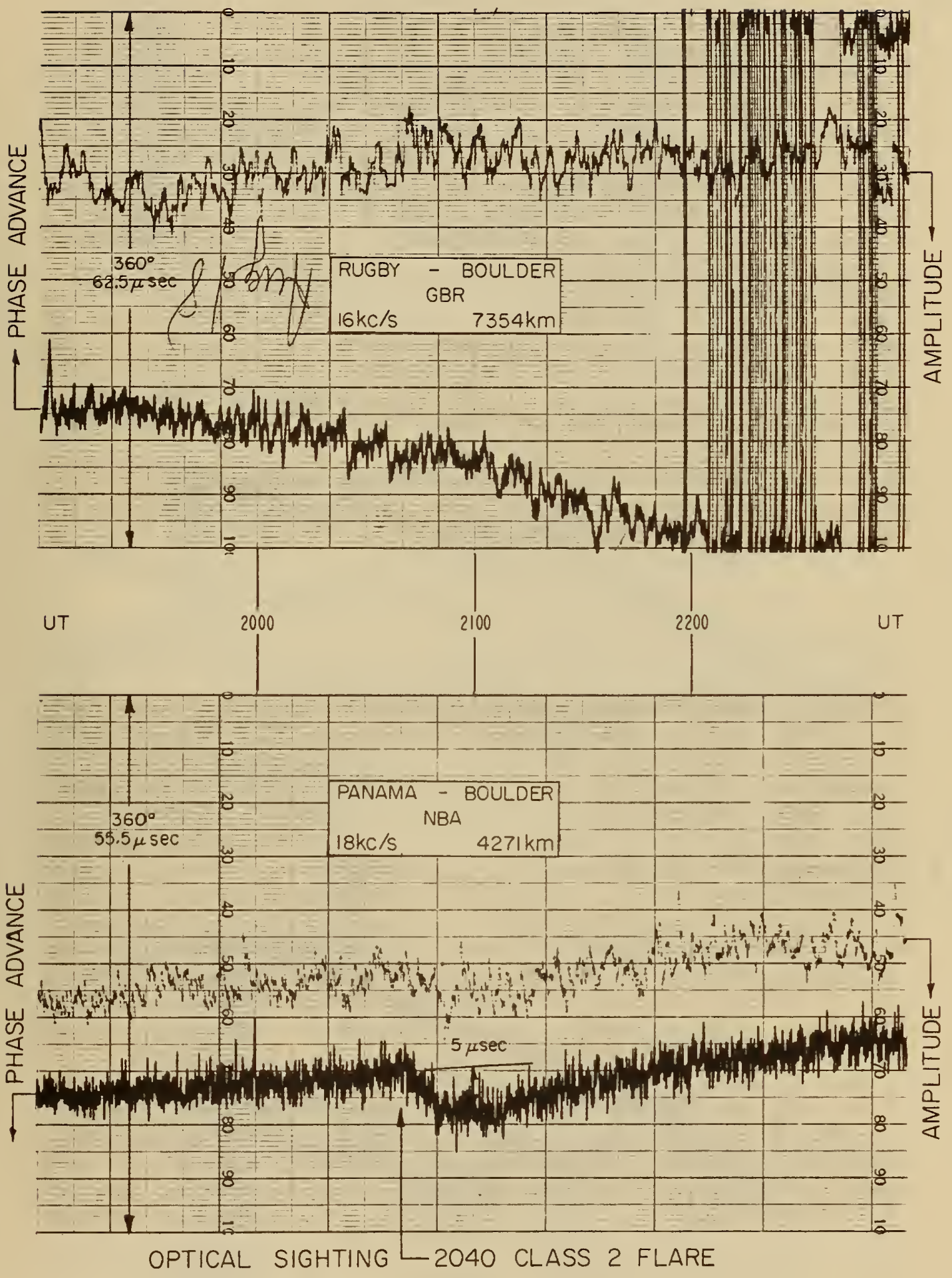

Figure 13 
SUDDEN PHASE ANOMALY I-SEPTEMBER 1961 UT
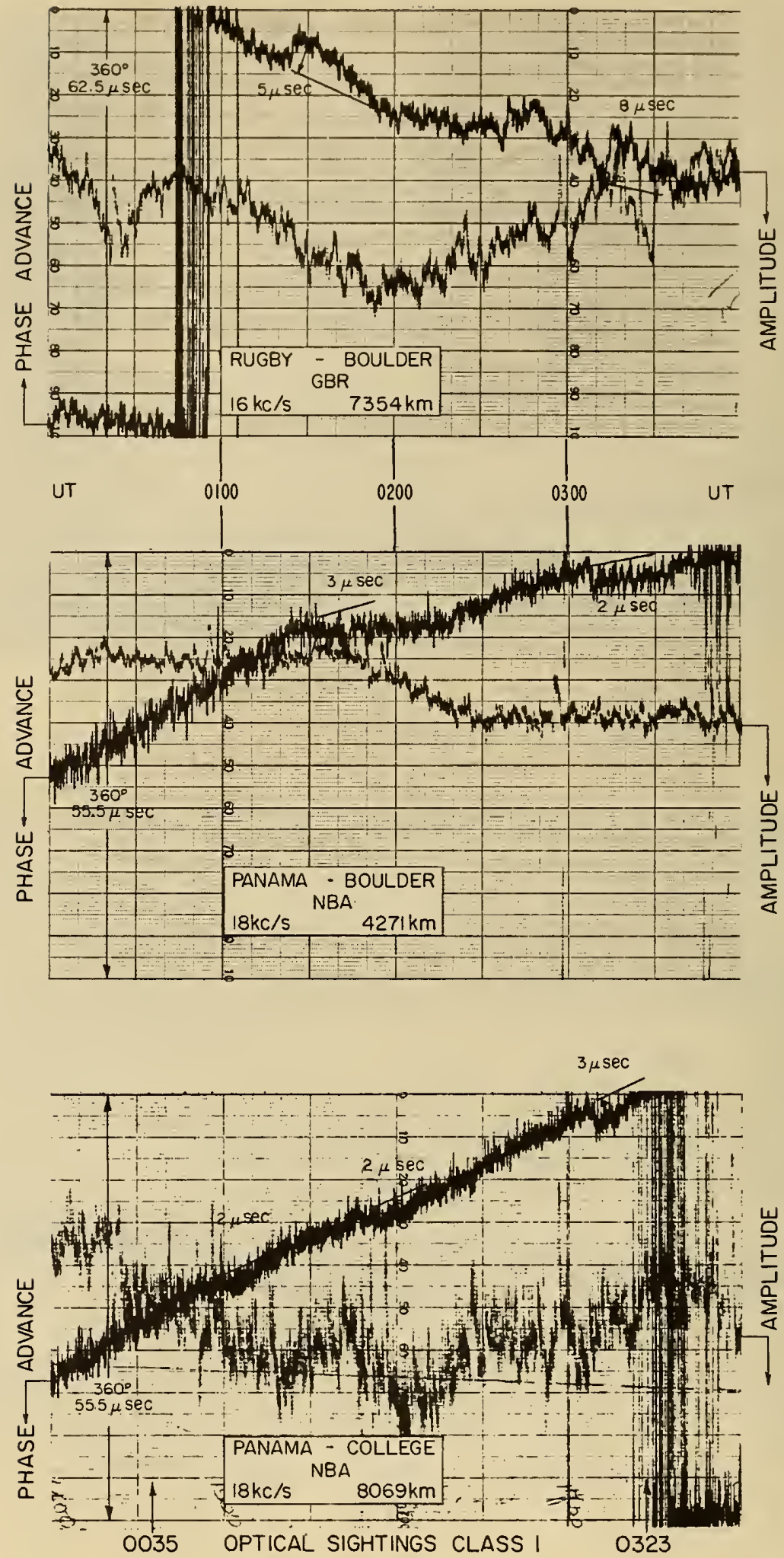

Figure 14 
SUDDEN PHASE ANOMALYS 2-SEPTEMBER 1961 UT
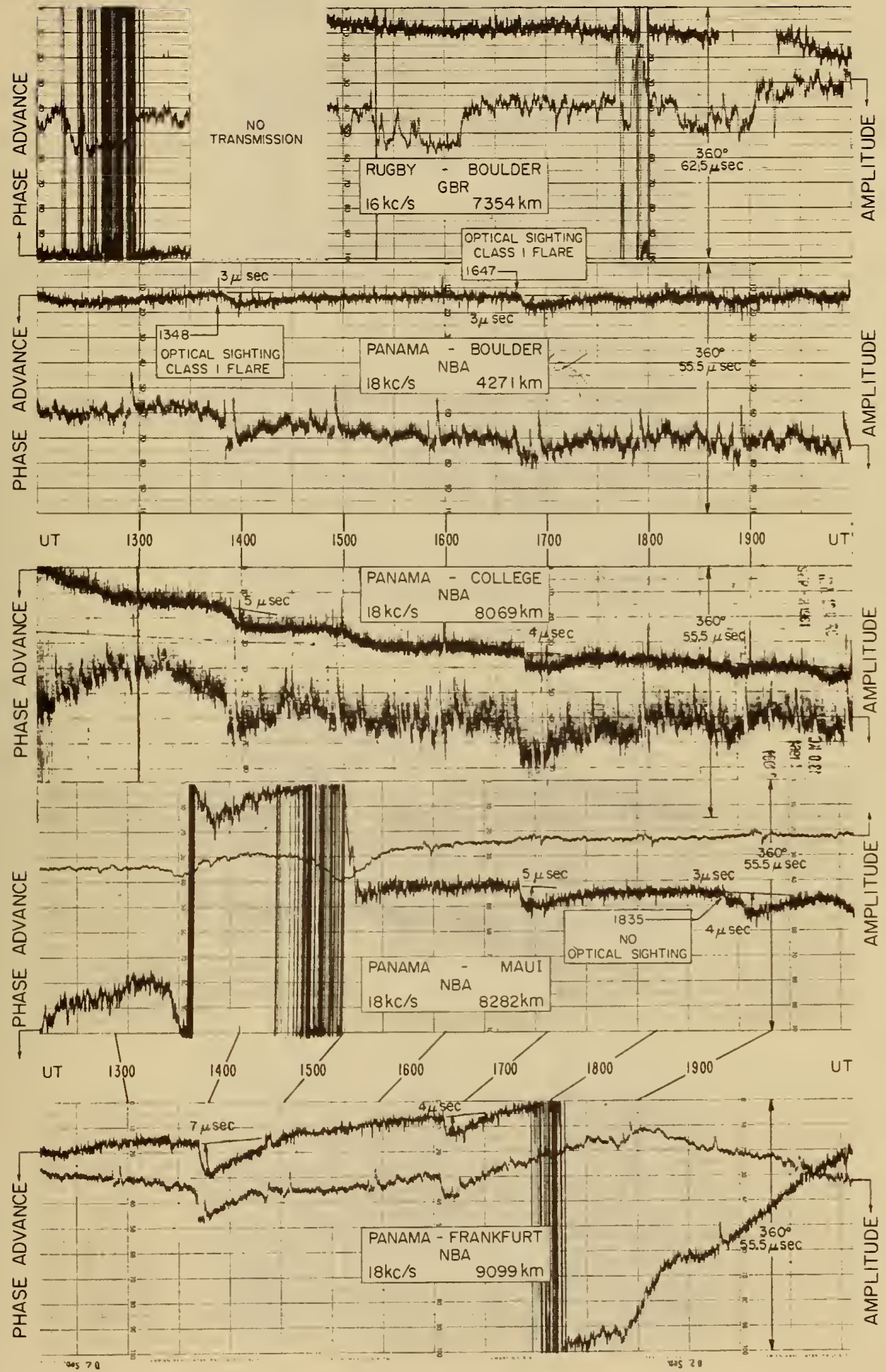

Figure 15 

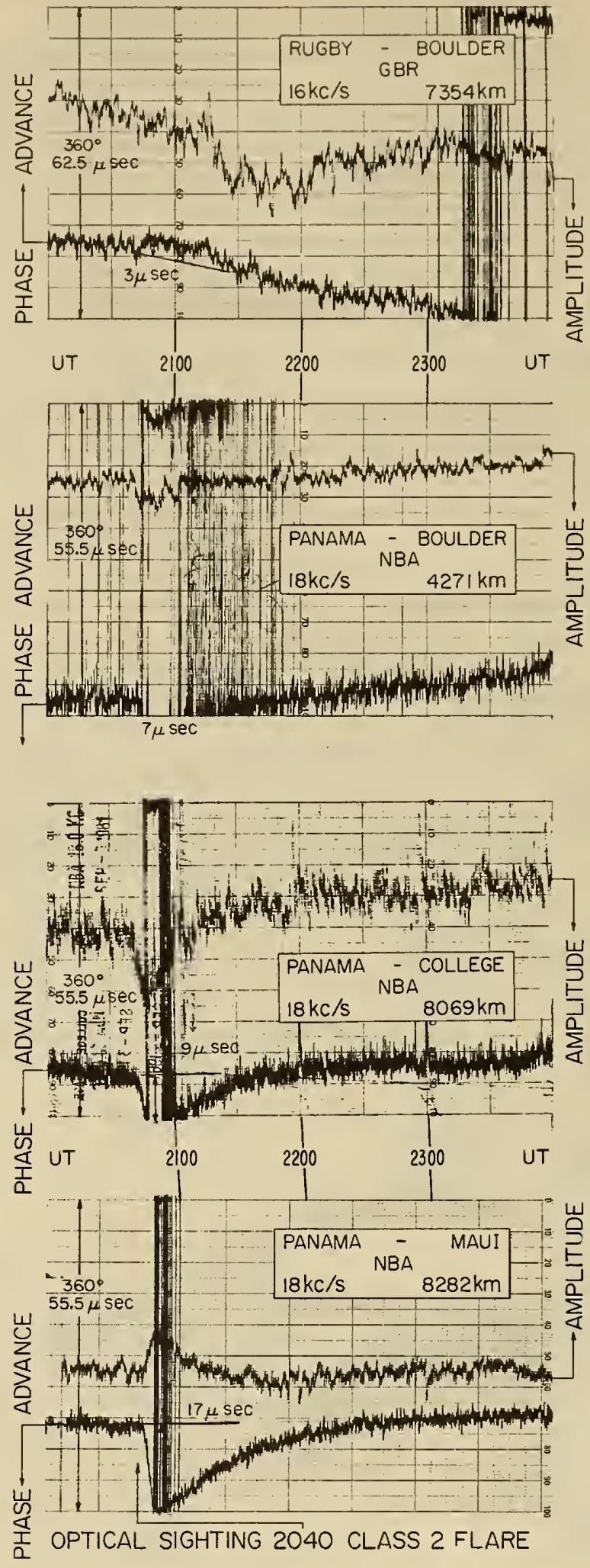

Figure 16 
SUDDEN PHASE ANOMALY 4-SEPTEMBER 1961 UT
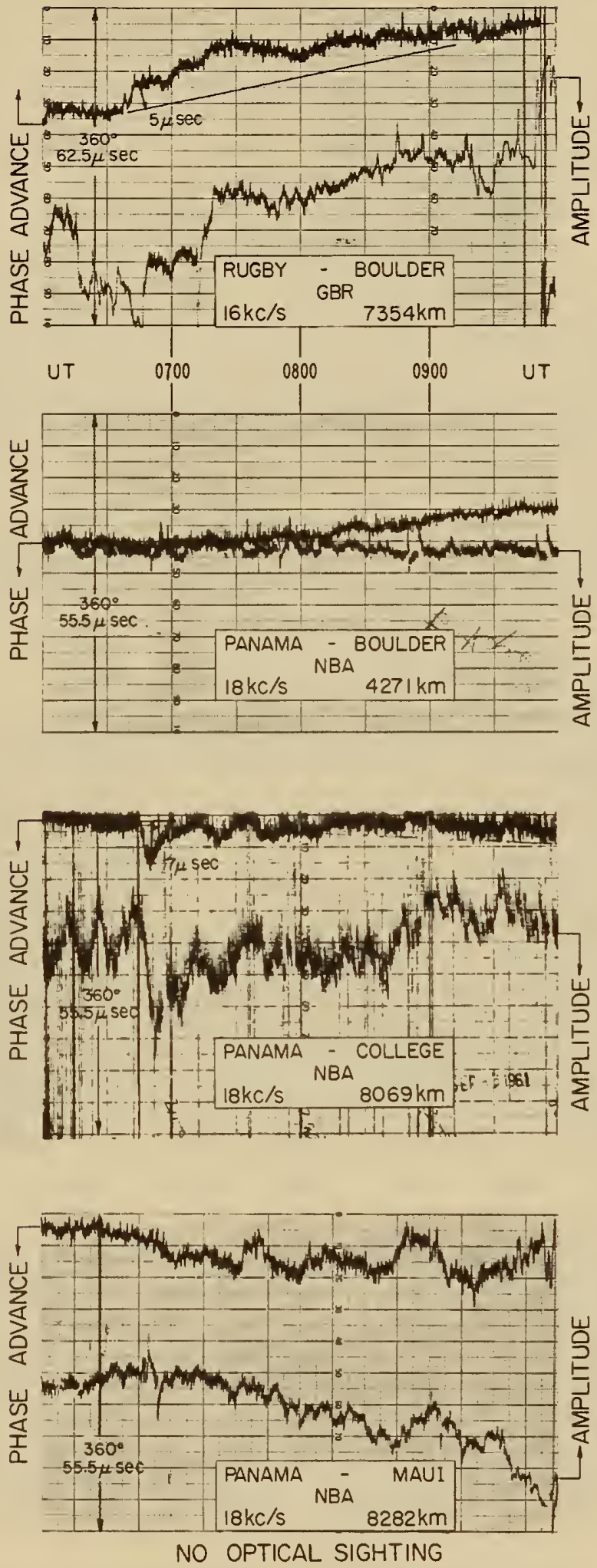

Figure 17a 


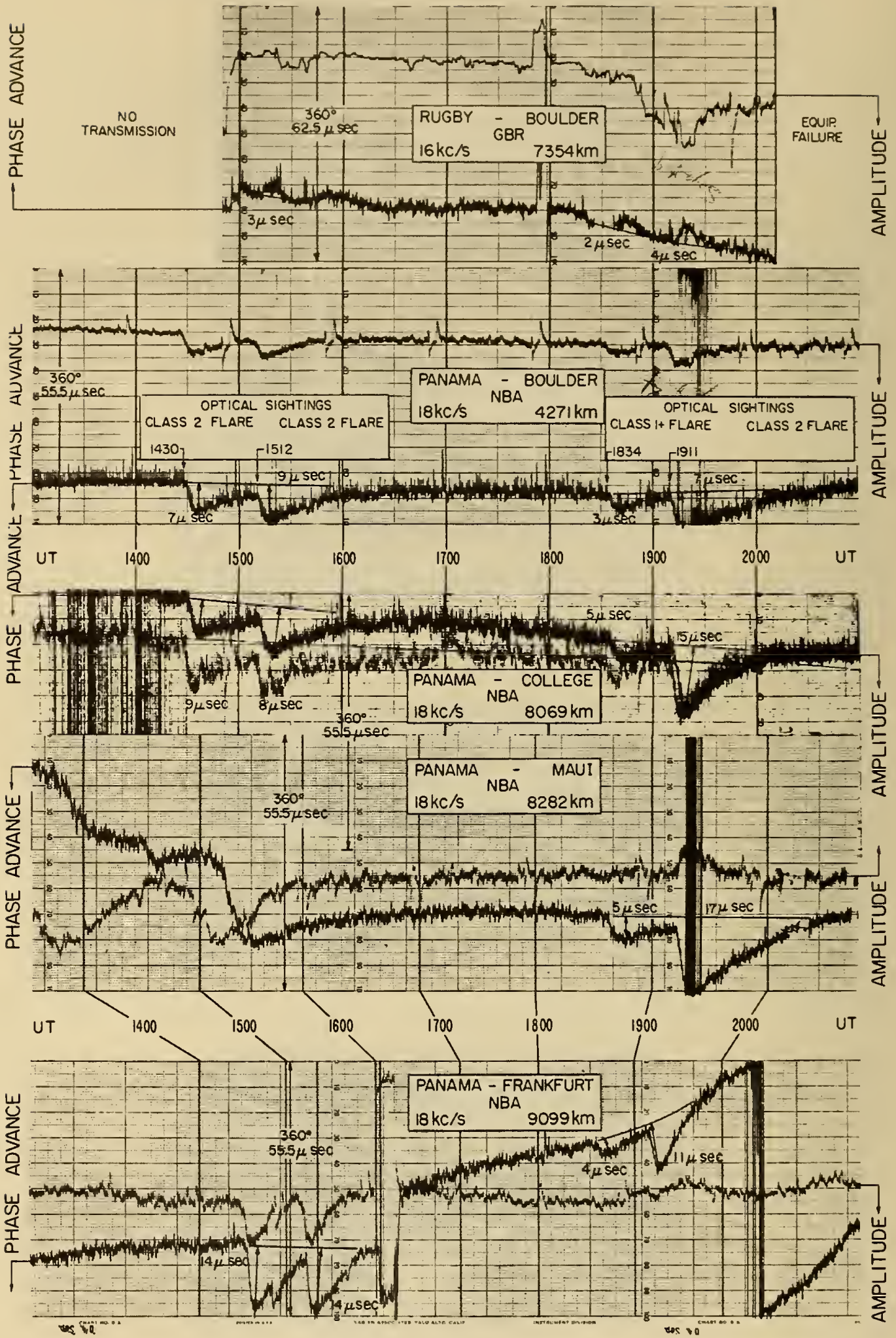

Multiple path SPA observations of 4 solar flares which occurred on 4 September 1961 UT.

Figure 17b 

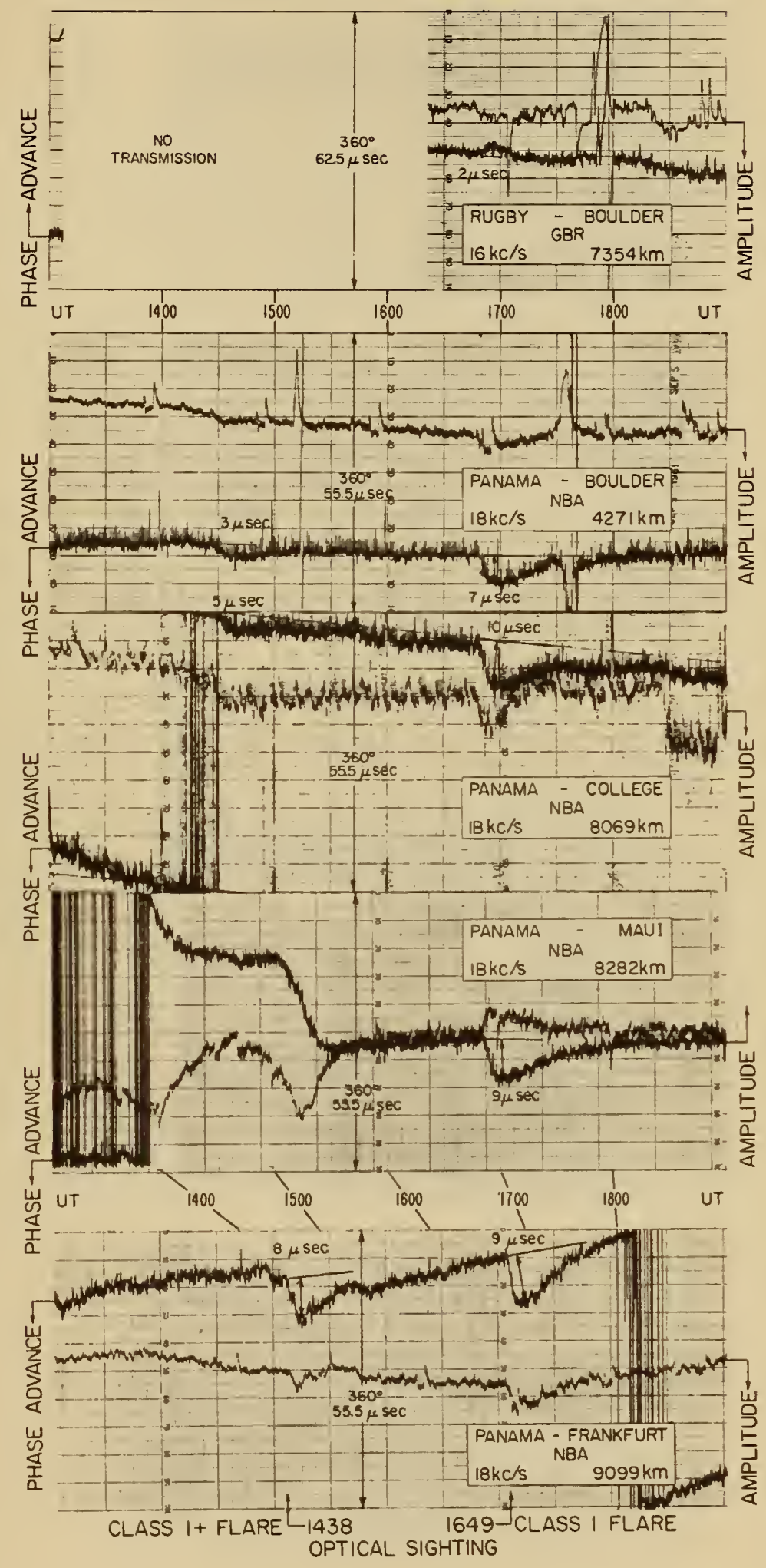

Figure 18 

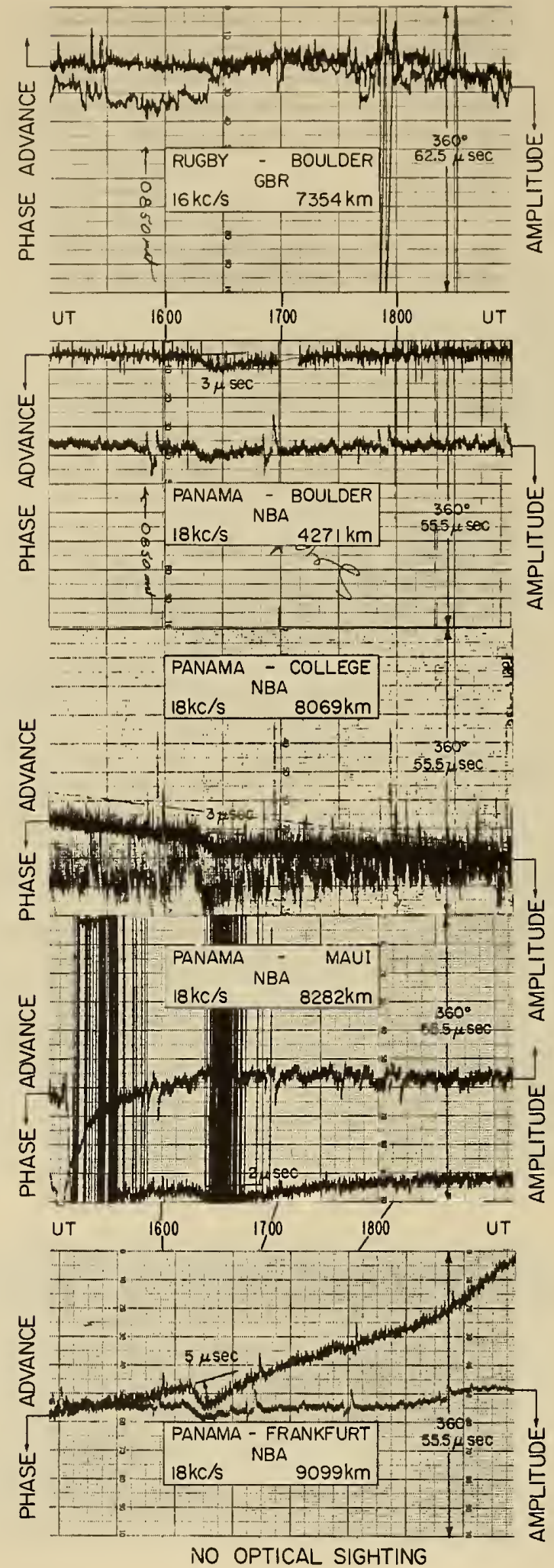

Figure 19 
SUDDEN PHASE ANOMALY 8-SEPTEMBER 1961 UT
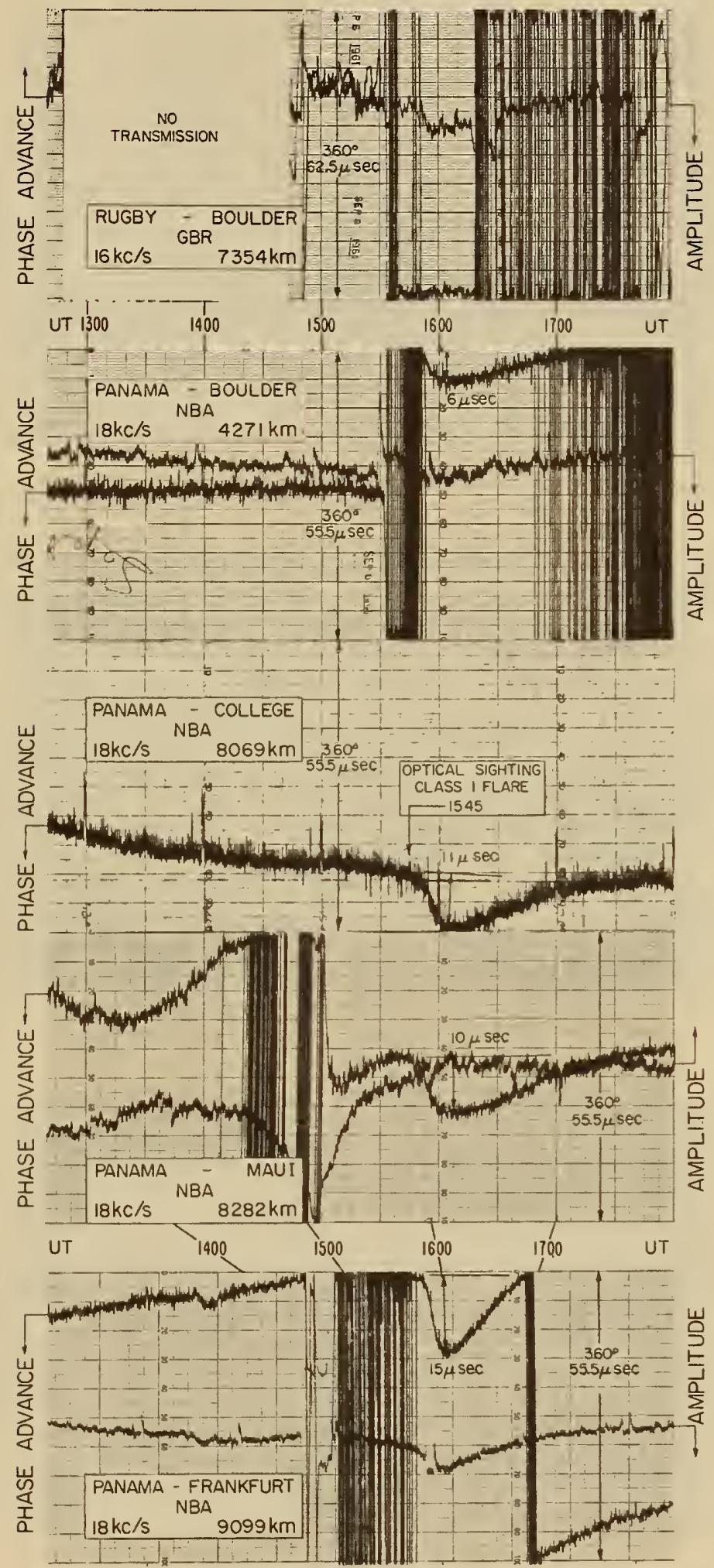

Figure 20 
SUDDEN PHASE ANOMALY 5-NOVEMBER 196I UT
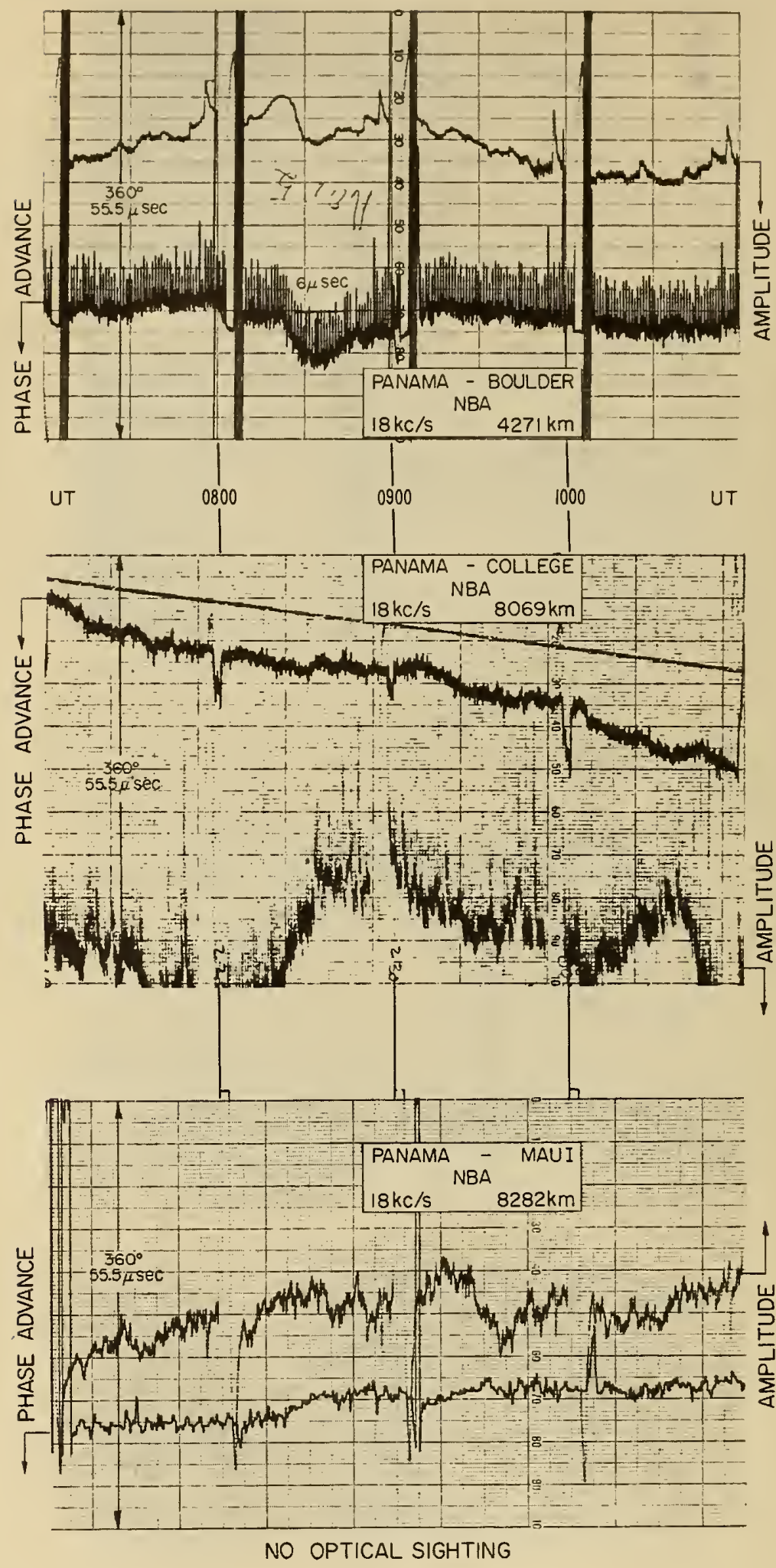

Figure 21 
SUDDEN PHASE ANOMALY IO-NOVEMBER I96| UT
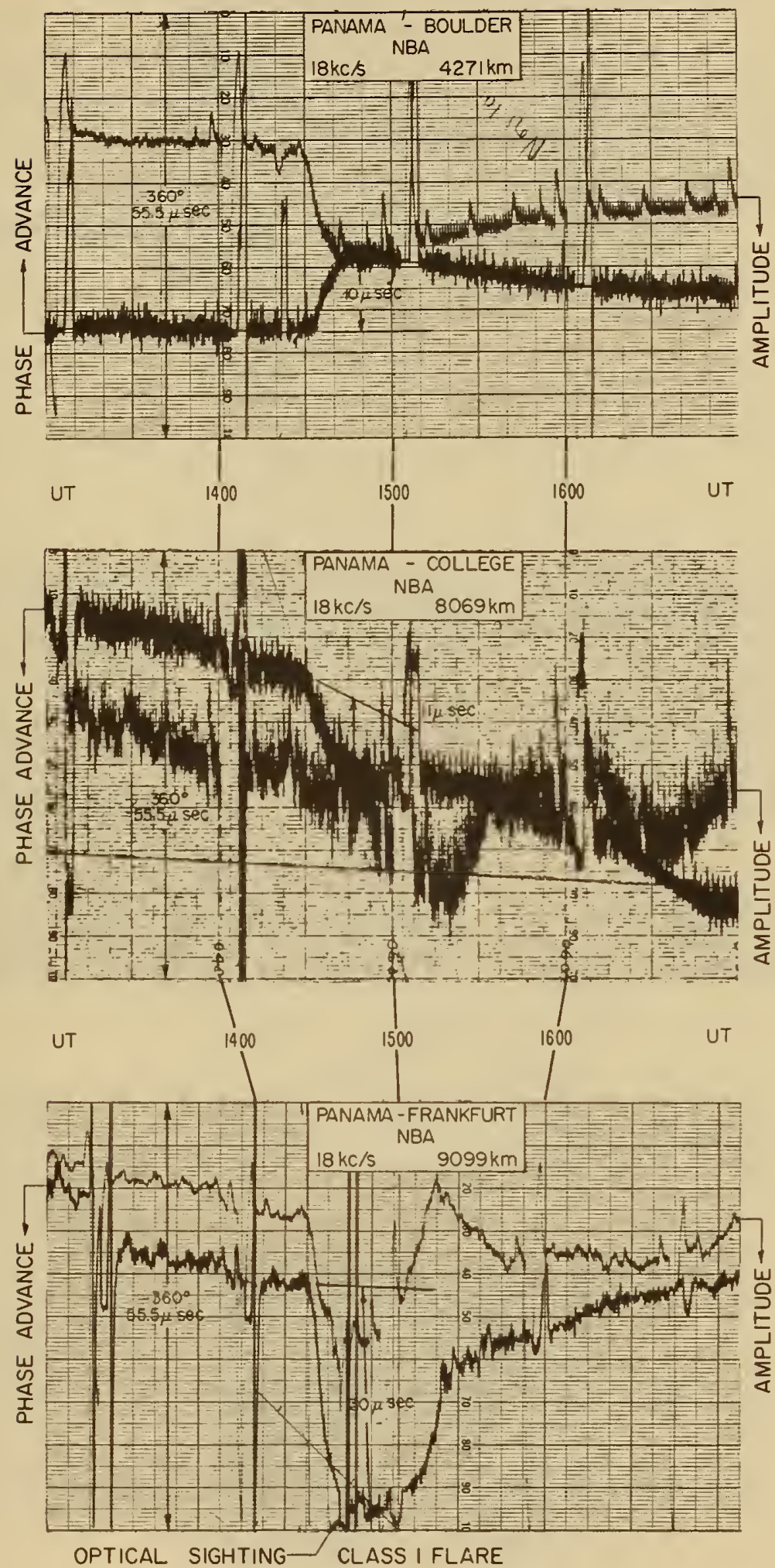

Figure 22 


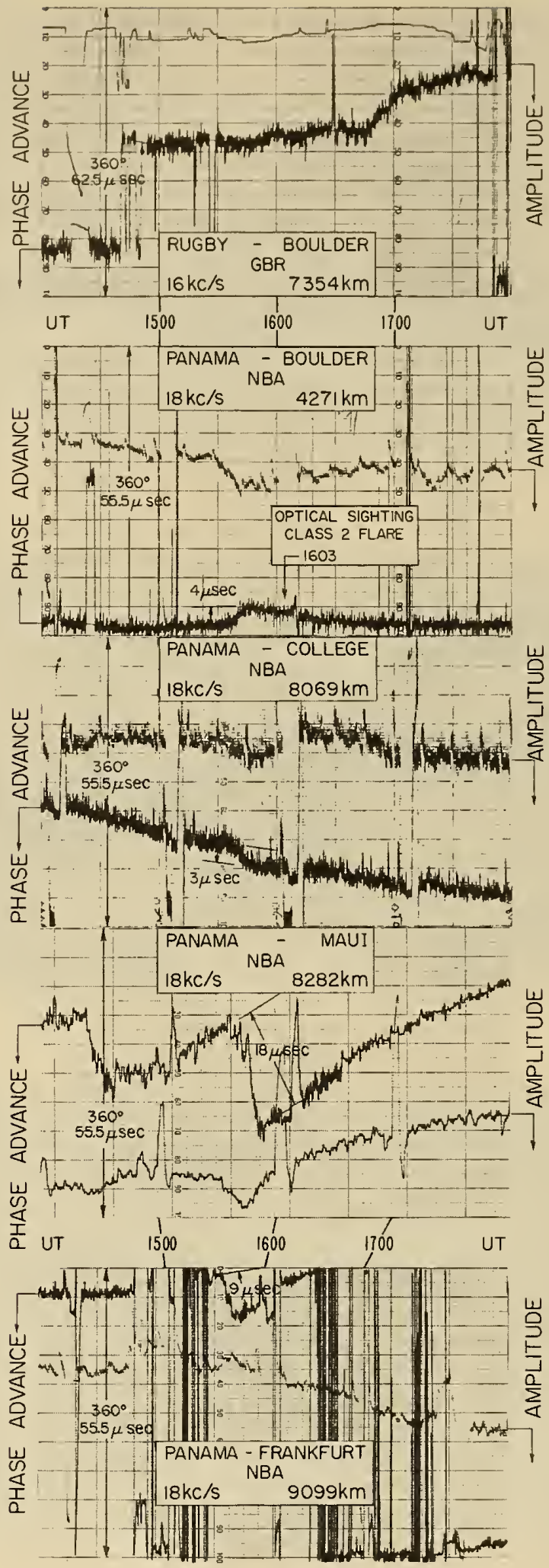

Figure 23 

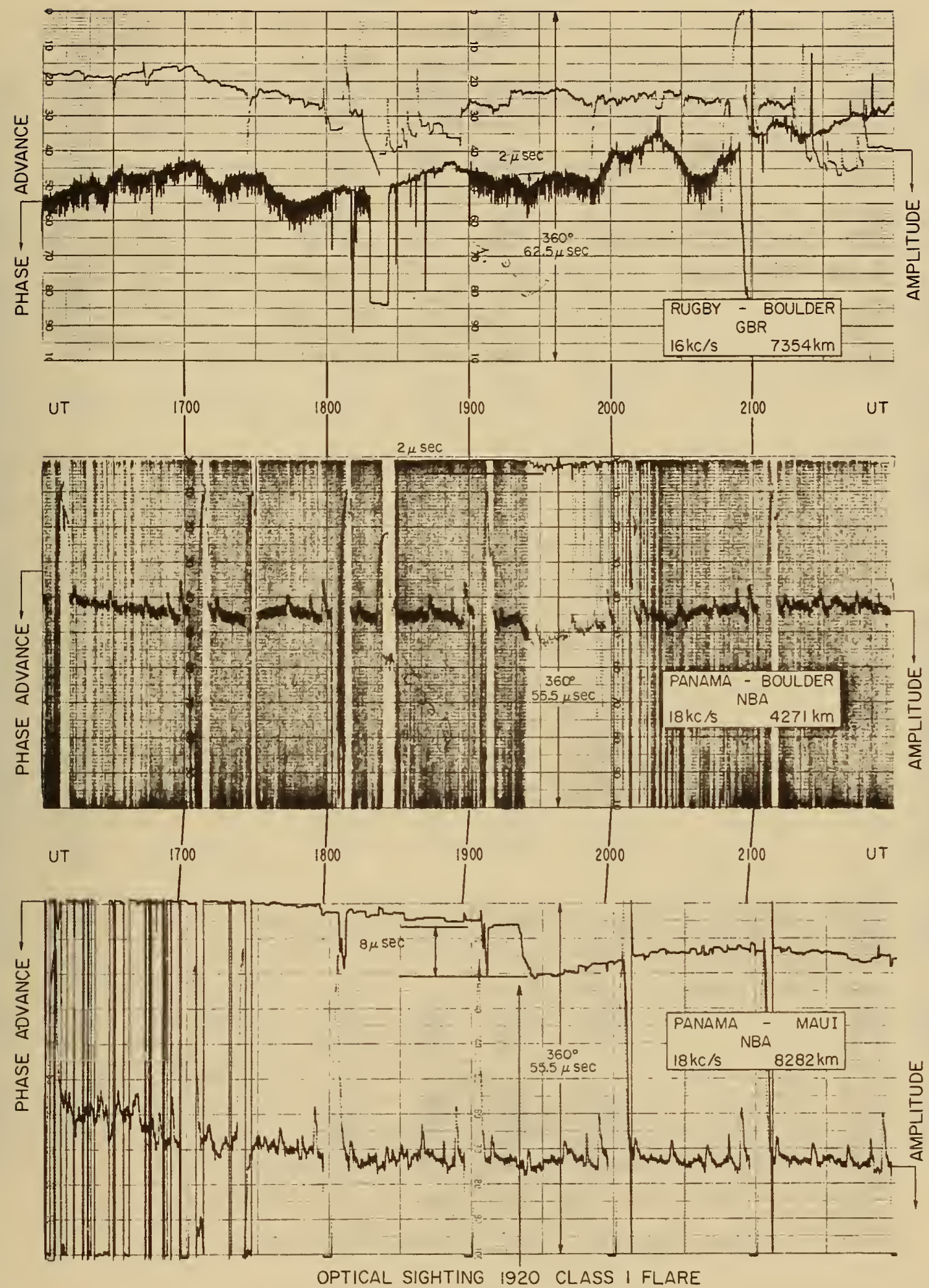

Figure 24 
TABLE No. 1

\begin{tabular}{|c|c|c|c|c|c|c|c|c|c|c|c|c|}
\hline $\begin{array}{l}\text { Flare } \\
\text { Number }\end{array}$ & $\begin{array}{l}\text { Date } \\
1961 \\
\end{array}$ & $\begin{array}{c}\text { Optical } \\
\text { Clas } 8 \\
\end{array}$ & $\begin{array}{c}\text { Optical } \\
\text { Sighting } \\
\text { UT } \\
\end{array}$ & $\begin{array}{l}\text { Time of } \\
\text { Beginning } \\
\text { SPA (UT) }\end{array}$ & $\begin{array}{l}\text { Time of } \\
\text { Maximum } \\
\text { SPA (UT) }\end{array}$ & $\begin{array}{l}\text { End Time } \\
\text { SPA (UT) }\end{array}$ & $\begin{array}{c}\Delta \varphi \\
\text { Degreea }\end{array}$ & $\begin{array}{c}\Delta \varphi \\
\text { Micro Sec }\end{array}$ & $\begin{array}{l}\Delta \mathrm{h} \\
\mathrm{Km}\end{array}$ & $\begin{array}{c}\frac{d p}{d t} \\
\text { Deg/Min }\end{array}$ & $\begin{array}{l}X \text { Ave. } \\
\text { Degrees }\end{array}$ & $\begin{array}{l}\text { Average } \\
\operatorname{Cos} X \\
\end{array}$ \\
\hline $\begin{array}{l}1 \\
\mathrm{NBA}-\mathrm{BO} \\
\mathrm{GBR}-\mathrm{BO}\end{array}$ & 1 May & 1 & 1621 & $\begin{array}{l}1623 \\
1623\end{array}$ & $\begin{array}{l}1630 \\
1634\end{array}$ & $\begin{array}{l}1852 \\
1745\end{array}$ & $\begin{array}{l}26 \\
29\end{array}$ & $\begin{array}{l}4 \\
5\end{array}$ & $\begin{array}{l}2.2 \\
1.4\end{array}$ & $\begin{array}{l}4 \\
3\end{array}$ & $\begin{array}{l}27.51 \\
47.35\end{array}$ & $\begin{array}{l}.88689 \\
.67747\end{array}$ \\
\hline $\begin{array}{l}2 \\
\text { NBA-BO } \\
\text { GBR-BO }\end{array}$ & 5 Jun & 1 & 1523 & $\begin{array}{l}1523 \\
1523\end{array}$ & $\begin{array}{l}1538 \\
1534\end{array}$ & $\begin{array}{l}1644 \\
1650\end{array}$ & $\begin{array}{l}39 \\
17\end{array}$ & $\begin{array}{l}6 \\
3\end{array}$ & $\begin{array}{l}3.3 \\
0.8\end{array}$ & $\begin{array}{l}4 \\
2\end{array}$ & $\begin{array}{l}38.23 \\
41.58\end{array}$ & $\begin{array}{l}.78550 \\
.74801\end{array}$ \\
\hline $\begin{array}{l}3 \\
N B A-B O \\
G B R-B O \\
\text { NBA-CO } \\
\text { NPG-CO }\end{array}$ & $11 \mathrm{Jul}$ & $1+$ & 1133 & 1135 & 1142 & 1225 & 32 & 5 & 2.7 & 9 & $\begin{array}{l}87.11 \\
61.22 \\
86.88 \\
90.00\end{array}$ & $\begin{array}{r}.05029 \\
.43133 \\
.05439 \\
0000\end{array}$ \\
\hline $\begin{array}{l}4 \\
\text { NBA-BO } \\
\text { NBA-CO } \\
\text { NPG-CO }\end{array}$ & $11 \mathrm{Ju} 1$ & $1+$ & 1332 & $\begin{array}{l}1334 \\
1335\end{array}$ & $\begin{array}{l}1340 \\
1340\end{array}$ & $\begin{array}{l}1500 \\
1450\end{array}$ & $\begin{array}{l}91 \\
39\end{array}$ & $\begin{array}{r}14 \\
6\end{array}$ & $\begin{array}{l}7.6 \\
1.7\end{array}$ & $\begin{array}{r}12 \\
7\end{array}$ & $\begin{array}{l}63.35 \\
67.81 \\
82.00\end{array}$ & $\begin{array}{r}.44843 \\
.37756 \\
.13913\end{array}$ \\
\hline $\begin{array}{l}5 \\
\text { GBR-BO } \\
N B A=C O \\
N B A-B O \\
\text { NPG-CO }\end{array}$ & $11 \mathrm{Ju} 1$ & 3 & 1640 & $\begin{array}{l}1636 \\
1634 \\
1616 \\
1633\end{array}$ & $\begin{array}{l}1712 \\
1710 \\
1710 \\
1711\end{array}$ & $\begin{array}{l}2000 \\
2100 \\
2000 \\
2100\end{array}$ & $\begin{array}{r}250 \\
168 \\
155 \\
47\end{array}$ & $\begin{array}{r}26 \\
26 \\
24 \\
7\end{array}$ & $\begin{array}{r}8.6 \\
8.5 \\
15.0\end{array}$ & $\begin{array}{r}8 \\
10 \\
7 \\
1\end{array}$ & $\begin{array}{l}41.36 \\
38.27 \\
24.41 \\
58.48\end{array}$ & $\begin{array}{r}.75050 \\
.78501 \\
.91058 \\
.52268\end{array}$ \\
\hline $\begin{array}{l}6 \\
\text { NBA-BO } \\
\text { GBR-BO }\end{array}$ & $15 \mathrm{Jul}$ & $2+$ & 1510 & $\begin{array}{l}1511 \\
1510\end{array}$ & $\begin{array}{l}1517 \\
1526\end{array}$ & & $\begin{array}{l}26 \\
17\end{array}$ & $\begin{array}{l}4 \\
3\end{array}$ & $\begin{array}{l}2.2 \\
0.8\end{array}$ & $\begin{array}{l}9 \\
2\end{array}$ & $\begin{array}{l}42.44 \\
43.23\end{array}$ & $\begin{array}{r}.73791 \\
.72861\end{array}$ \\
\hline $\begin{array}{l}7 \\
\text { NBA-BO } \\
\text { GBR-BO } \\
\text { NBA-CO } \\
\text { NPG-CO }\end{array}$ & $17 \mathrm{Ju} 1$ & No Opt & al Sighting & $\begin{array}{l}2007 \\
1951 \\
1950\end{array}$ & $\begin{array}{l}2015 \\
2012 \\
2012\end{array}$ & $\begin{array}{l}2120 \\
2100 \\
2112\end{array}$ & $\begin{array}{l}19 \\
19 \\
13\end{array}$ & $\begin{array}{l}3 \\
3 \\
2\end{array}$ & $\begin{array}{l}1.6 \\
0.9\end{array}$ & $\begin{array}{r}10 \\
1 \\
2\end{array}$ & $\begin{array}{l}29.33 \\
57.92 \\
34.05 \\
37.98\end{array}$ & $\begin{array}{l}.87173 \\
.53104 \\
.82847 \\
.78815\end{array}$ \\
\hline $\begin{array}{l}8 \\
\text { NBA-BO } \\
\text { GBR-BO } \\
\text { NBA-CO } \\
\text { NPG-CO }\end{array}$ & $17 \mathrm{Jul}$ & 1 & 2125 & $\begin{array}{l}2140 \\
2140 \\
2140\end{array}$ & $\begin{array}{l}2147 \\
2210 \\
2148\end{array}$ & $\begin{array}{l}2330 \\
2342 \\
2348\end{array}$ & $\begin{array}{l}39 \\
29 \\
65\end{array}$ & $\begin{array}{r}6 \\
5 \\
10\end{array}$ & $\begin{array}{l}3.3 \\
1.4 \\
2.8\end{array}$ & $\begin{array}{r}40 \\
1 \\
14\end{array}$ & $\begin{array}{l}49.20 \\
63.67 \\
46.77 \\
37.77\end{array}$ & $\begin{array}{l}.65335 \\
.44352 \\
.68492 \\
.79048\end{array}$ \\
\hline $\begin{array}{l}9 \\
\mathrm{NBA}-\mathrm{BO} \\
\mathrm{GBR}-\mathrm{BO} \\
\mathrm{NBA}-\mathrm{CO} \\
\mathrm{NPG}-\mathrm{CO}\end{array}$ & $18 \mathrm{Ju} 1$ & 2 & 0813 & 0813 & 0815 & & 19 & 3 & 0.9 & 13 & $\begin{array}{l}90.00 \\
72.37 \\
90.00 \\
90.00\end{array}$ & $\begin{array}{r}0000 \\
.30283 \\
0000 \\
0000\end{array}$ \\
\hline $\begin{array}{l}10 \\
\text { NBA-BO } \\
\text { GBR-BO } \\
\text { NBA-CO } \\
\text { NPG-CO }\end{array}$ & $18 \mathrm{Ju} 1$ & 3 & 0945 & 0956 & 1006 & 1035 & 40 & 7 & 2.0 & 4 & $\begin{array}{l}90.00 \\
66.10 \\
90.00 \\
90.00\end{array}$ & $\begin{array}{r}0000 \\
.40498 \\
0000 \\
0000\end{array}$ \\
\hline $\begin{array}{l}11 \\
\text { GBR-BO } \\
\text { NBA-BO } \\
\text { NPG-CO }\end{array}$ & $20 \mathrm{Jul}$ & 3 & 1553 & $\begin{array}{l}1553 \\
1553 \\
1553\end{array}$ & $\begin{array}{l}1557 \\
1600 \\
1557\end{array}$ & $\begin{array}{l}1950 \\
2000 \\
2000\end{array}$ & $\begin{array}{r}86 \\
110 \\
33\end{array}$ & $\begin{array}{r}15 \\
17 \\
5\end{array}$ & $\begin{array}{r}5.0 \\
10.6\end{array}$ & $\begin{array}{l}30 \\
90 \\
30\end{array}$ & $\begin{array}{l}42.70 \\
34.18 \\
65.74\end{array}$ & $\begin{array}{r}.73491 \\
.82724 \\
.41078\end{array}$ \\
\hline $\begin{array}{l}12 \\
\text { GBR-BO } \\
\text { NBA-BO } \\
\text { NBA-CO } \\
\text { NPG-CO }\end{array}$ & $21 \mathrm{Ju} 1$ & 2 & 1714 & $\begin{array}{l}1702 \\
1702 \\
1703\end{array}$ & $\begin{array}{l}1710 \\
1710 \\
1712\end{array}$ & $\begin{array}{l}2000 \\
2000 \\
1753\end{array}$ & $\begin{array}{l}64 \\
78 \\
13\end{array}$ & $\begin{array}{r}10 \\
12 \\
2\end{array}$ & $\begin{array}{l}6.2 \\
3.9\end{array}$ & $\begin{array}{r}42 \\
22 \\
1\end{array}$ & $\begin{array}{l}43.41 \\
19.86 \\
35.94 \\
56.45\end{array}$ & $\begin{array}{l}.72635 \\
.94050 \\
.80960 \\
.55264\end{array}$ \\
\hline
\end{tabular}


TABLE No. 1 (page 2)

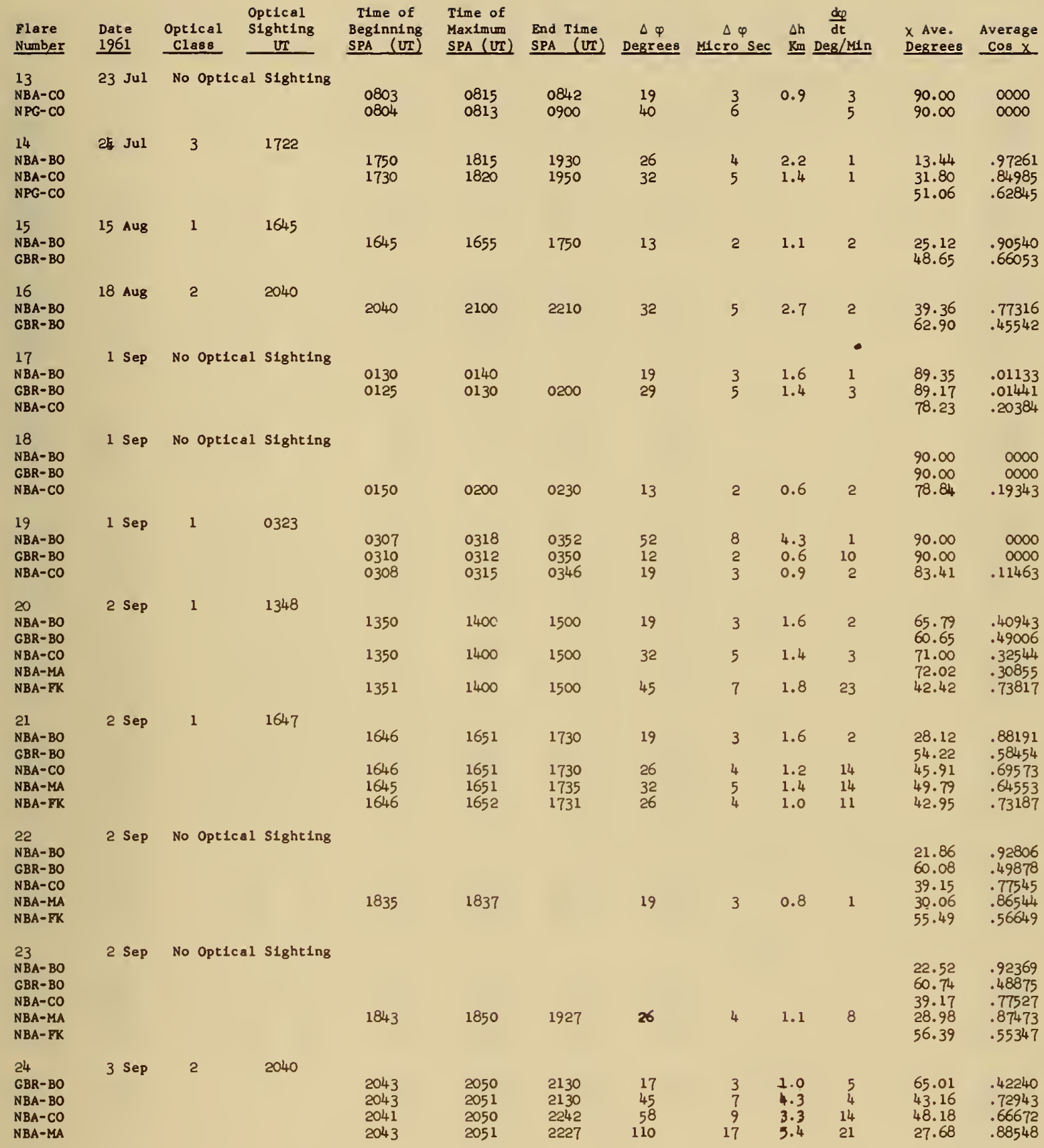


TABLE No. 1 (page 3)

\begin{tabular}{|c|c|c|c|c|c|c|c|c|c|c|c|c|}
\hline $\begin{array}{l}\text { Flare } \\
\text { Number }\end{array}$ & $\begin{array}{l}\text { Date } \\
1961 \\
\end{array}$ & $\begin{array}{l}\text { Optical } \\
\text { Class } \\
\end{array}$ & $\begin{array}{l}\text { ptical } \\
\text { Ighting } \\
\text { UT } \\
\end{array}$ & $\begin{array}{l}\text { Time of } \\
\text { Beginning } \\
\text { SPA (UT) }\end{array}$ & $\begin{array}{l}\text { Ilme of } \\
\text { Maximum } \\
\text { SPA (UT) } \\
\end{array}$ & $\begin{array}{l}\text { End } T \text { ime } \\
\text { SPA (UI) }\end{array}$ & $\begin{array}{c}\Delta \varphi \\
\text { Degrees } \\
\end{array}$ & $\begin{array}{c}\Delta \varphi \\
\text { Micro Sec }\end{array}$ & $\begin{array}{l}\Delta \mathrm{h} \\
\mathrm{Km}\end{array}$ & $\begin{array}{c}\frac{d \varphi}{d t} \\
\text { Deg/M1n }\end{array}$ & $\begin{array}{l}\text { X Ave. } \\
\text { Degrees }\end{array}$ & $\begin{array}{l}\text { Average } \\
\operatorname{Cos} x \\
\end{array}$ \\
\hline $\begin{array}{l}25 \\
N B A-B O \\
G B R-B O \\
N B A-C O \\
\text { NBA-MA }\end{array}$ & 4 Sep & No Optical & S1ghting & $\begin{array}{l}0637 \\
0645\end{array}$ & $\begin{array}{l}0645 \\
0650\end{array}$ & & $\begin{array}{l}29 \\
45\end{array}$ & $\begin{array}{l}5 \\
7\end{array}$ & $\begin{array}{l}1.4 \\
2.0\end{array}$ & $\begin{array}{r}9 \\
13\end{array}$ & $\begin{array}{l}90.00 \\
84.52 \\
90.00 \\
90.00\end{array}$ & $\begin{array}{r}0000 \\
.09538 \\
0000 \\
0000\end{array}$ \\
\hline $\begin{array}{l}26 \\
\text { NBA-BO } \\
\text { NBA-CO } \\
\text { NBA-MA } \\
\text { NBA-FK }\end{array}$ & 4 Sep & 2 & 1430 & $\begin{array}{l}1429 \\
1431 \\
1430\end{array}$ & $\begin{array}{l}1437 \\
1436 \\
1438\end{array}$ & & $\begin{array}{l}45 \\
58 \\
91\end{array}$ & $\begin{array}{r}7 \\
9 \\
14\end{array}$ & $\begin{array}{l}3.8 \\
2.6 \\
3.6\end{array}$ & $\begin{array}{l}10 \\
24 \\
23\end{array}$ & $\begin{array}{l}54.59 \\
64.55 \\
66.06 \\
38.98\end{array}$ & $\begin{array}{l}.57940 \\
.42972 \\
.40576 \\
.77731\end{array}$ \\
\hline $\begin{array}{l}27 \\
\text { NBA-BO } \\
\text { GBR-BO } \\
\text { NBA-CO } \\
\text { NBA-MA } \\
\text { NBA-FK }\end{array}$ & 4 Sep & 2 & 1512 & $\begin{array}{l}1510 \\
1510 \\
1512 \\
1514\end{array}$ & $\begin{array}{l}1518 \\
1518 \\
1520 \\
1520\end{array}$ & $\begin{array}{l}1650 \\
1532 \\
1641 \\
1615\end{array}$ & $\begin{array}{l}58 \\
17 \\
52 \\
91\end{array}$ & $\begin{array}{r}9 \\
3 \\
8 \\
14\end{array}$ & $\begin{array}{l}4.9 \\
0.9 \\
2.3 \\
3.6\end{array}$ & $\begin{array}{r}16 \\
2 \\
10 \\
25\end{array}$ & $\begin{array}{l}45.88 \\
55.54 \\
58.97 \\
61.72 \\
38.12\end{array}$ & $\begin{array}{r}.69612 \\
.56575 \\
.51538 \\
.47364 \\
.78661\end{array}$ \\
\hline $\begin{array}{l}28 \\
\text { GBR-BO } \\
\text { NBA-BO } \\
\text { NBA-CO } \\
\text { NBA-MA } \\
\text { NBA-FK }\end{array}$ & 4 Sep & 1 & 1834 & $\begin{array}{l}1833 \\
1831 \\
1837 \\
1837 \\
1836\end{array}$ & $\begin{array}{l}1843 \\
1841 \\
1844 \\
1846 \\
1845\end{array}$ & 1900 & $\begin{array}{l}11 \\
19 \\
32 \\
32 \\
25\end{array}$ & $\begin{array}{l}2 \\
3 \\
5 \\
5 \\
4\end{array}$ & $\begin{array}{l}1.0 \\
1.8 \\
1.6 \\
1.9 \\
1.1\end{array}$ & $\begin{array}{r}1 \\
5 \\
5 \\
27 \\
3\end{array}$ & $\begin{array}{l}61.01 \\
22.73 \\
39.75 \\
29.84 \\
56.08\end{array}$ & $\begin{array}{r}.48463 \\
.92233 \\
.76878 \\
.86740 \\
.55803\end{array}$ \\
\hline $\begin{array}{l}29 \\
\text { GBR-BO } \\
\text { NBA-BO } \\
\text { NBA-CO } \\
\text { NBA-MA } \\
\text { NBA-FK }\end{array}$ & 4 Sep & 2 & 1911 & $\begin{array}{l}1910 \\
1910 \\
1912 \\
1913 \\
1910\end{array}$ & $\begin{array}{l}1919 \\
1917 \\
1919 \\
1921 \\
1917\end{array}$ & $\begin{array}{l}1940 \\
2045 \\
2020 \\
2027 \\
1943\end{array}$ & $\begin{array}{r}23 \\
45 \\
97 \\
110 \\
71\end{array}$ & $\begin{array}{r}4 \\
7 \\
15 \\
17 \\
11\end{array}$ & $\begin{array}{l}1.3 \\
5.0 \\
4.6 \\
5.4 \\
2.5\end{array}$ & $\begin{array}{r}4 \\
10 \\
18 \\
18 \\
12\end{array}$ & $\begin{array}{l}61.60 \\
26.85 \\
40.56 \\
25.96 \\
59.32\end{array}$ & $\begin{array}{r}.47560 \\
.89219 \\
.75964 \\
.89908 \\
.51016\end{array}$ \\
\hline $\begin{array}{l}30 \\
\text { NBA-BO } \\
\text { NBA-CO } \\
\text { NBA-MA } \\
\text { NBA-FK }\end{array}$ & 5 sep & $1+$ & 1438 & $\begin{array}{l}1430 \\
1430 \\
1439\end{array}$ & $\begin{array}{l}1440 \\
1440 \\
1446\end{array}$ & $\begin{array}{l}1510 \\
1520 \\
1520\end{array}$ & $\begin{array}{l}19 \\
32 \\
52\end{array}$ & $\begin{array}{l}3 \\
5 \\
8\end{array}$ & $\begin{array}{l}1.6 \\
1.4 \\
2.0\end{array}$ & $\begin{array}{l}2 \\
3 \\
6\end{array}$ & $\begin{array}{l}54.68 \\
64.38 \\
66.07 \\
39.25\end{array}$ & $\begin{array}{l}.57807 \\
.43239 \\
.40548 \\
.77432\end{array}$ \\
\hline $\begin{array}{l}31 \\
\text { NBA-BO } \\
\text { GBR-BO } \\
\text { NBA-CO } \\
\text { NBA-MA } \\
\text { NBA-FK }\end{array}$ & $5 \mathrm{Sep}$ & 1 & 1649 & $\begin{array}{l}1650 \\
1650 \\
1650 \\
1651 \\
1649\end{array}$ & $\begin{array}{l}1657 \\
1657 \\
1656 \\
1702 \\
1700\end{array}$ & $\begin{array}{l}1800 \\
1710 \\
1825 \\
1823 \\
1810\end{array}$ & $\begin{array}{l}45 \\
12 \\
65 \\
58 \\
58\end{array}$ & $\begin{array}{r}7 \\
2 \\
10 \\
9 \\
9\end{array}$ & $\begin{array}{l}3.8 \\
0.6 \\
2.9 \\
2.5 \\
2.3\end{array}$ & $\begin{array}{r}7 \\
2 \\
10 \\
10 \\
14\end{array}$ & $\begin{array}{l}27.64 \\
55.43 \\
45.88 \\
48.49 \\
44.58\end{array}$ & $\begin{array}{r}.88582 \\
.56734 \\
.69614 \\
.66263 \\
.71225\end{array}$ \\
\hline $\begin{array}{l}32 \\
\text { GBR-BO } \\
\text { NBA-BO } \\
\text { NBA-CO } \\
\text { NBA-MA } \\
\text { NBA-FK }\end{array}$ & $7 \mathrm{sep}$ & No Opt1cal & S1ght1ng & $\begin{array}{l}1618 \\
1618 \\
1620 \\
1620\end{array}$ & $\begin{array}{l}1628 \\
1628 \\
1632 \\
1626\end{array}$ & $\begin{array}{l}1730 \\
1730 \\
1722 \\
1729\end{array}$ & $\begin{array}{l}19 \\
19 \\
13 \\
32\end{array}$ & $\begin{array}{l}3 \\
3 \\
2 \\
5\end{array}$ & $\begin{array}{l}1.8 \\
0.9 \\
0.6 \\
1.4\end{array}$ & $\begin{array}{l}2 \\
2 \\
1 \\
4\end{array}$ & $\begin{array}{l}55.57 \\
33.39 \\
50.17 \\
55.39 \\
41.97\end{array}$ & $\begin{array}{r}.56542 \\
.83491 \\
.64038 \\
.56795 \\
.74347\end{array}$ \\
\hline $\begin{array}{l}33 \\
\text { GBR-BO } \\
\text { NBA-BO } \\
\text { NBA-CO } \\
\text { NBA-MA } \\
\text { NBA-FK }\end{array}$ & $8 \mathrm{sep}$ & 1 & 1545 & $\begin{array}{l}1531 \\
1551 \\
1547 \\
1542\end{array}$ & $\begin{array}{l}1603 \\
1605 \\
1611 \\
1602\end{array}$ & $\begin{array}{l}1730 \\
1723 \\
1745 \\
1742\end{array}$ & $\begin{array}{l}39 \\
71 \\
65 \\
97\end{array}$ & $\begin{array}{r}6 \\
11 \\
10 \\
15\end{array}$ & $\begin{array}{l}4.3 \\
3.6 \\
3.2 \\
4.4\end{array}$ & $\begin{array}{r}6 \\
6 \\
8 \\
12\end{array}$ & $\begin{array}{l}55.96 \\
38.53 \\
53.99 \\
57.88 \\
40.44\end{array}$ & $\begin{array}{r}.55973 \\
.78227 \\
.58789 \\
.53155 \\
.76102\end{array}$ \\
\hline $\begin{array}{l}34 \\
\text { NBA-BO } \\
\text { NBA-CO } \\
\text { NBA-MA }\end{array}$ & 5 Nov & No Optical & S1ght1ng & 0822 & 0833 & 0920 & 39 & 6 & $3 \cdot 3$ & 4 & $\begin{array}{l}90.00 \\
90.00 \\
90.00\end{array}$ & $\begin{array}{l}\infty 000 \\
0000 \\
0000\end{array}$ \\
\hline
\end{tabular}


TABLE No. 1 (page 4)

\begin{tabular}{|c|c|c|c|c|c|c|c|c|c|c|c|c|}
\hline $\begin{array}{l}\text { Plare } \\
\text { Number }\end{array}$ & $\begin{array}{l}\text { Date } \\
1961 \\
\end{array}$ & $\begin{array}{c}\text { Optical } \\
\text { Class } \\
\end{array}$ & $\begin{array}{l}\text { Optical } \\
\text { S1ghting } \\
\text { UT } \\
\end{array}$ & $\begin{array}{l}\text { Time of } \\
\text { Beginning } \\
\text { SPA (UT) }\end{array}$ & $\begin{array}{l}\text { Time of } \\
\text { Maximum } \\
\text { SPA (UT) } \\
\end{array}$ & $\begin{array}{l}\text { End Time } \\
\text { SPA (UI) }\end{array}$ & $\begin{array}{c}\Delta \varphi \\
\text { Degrees } \\
\end{array}$ & $\begin{array}{c}\Delta \varphi \\
\text { Micro Sec }\end{array}$ & $\begin{array}{l}\Delta \mathrm{h} \\
\mathrm{km}\end{array}$ & $\begin{array}{c}\frac{d \varphi}{d t} \\
\operatorname{Deg} / M i n\end{array}$ & $\begin{array}{l}X \text { Ave. } \\
\text { Degrees }\end{array}$ & $\begin{array}{l}\text { Average } \\
\cos x \\
\end{array}$ \\
\hline $\begin{array}{l}35 \\
N B A-B O \\
N B A-C O \\
N B A-B K\end{array}$ & 10 Hov & 1 & 1434 & $\begin{array}{l}1434 \\
1434 \\
1423\end{array}$ & $\begin{array}{l}1449 \\
1446 \\
1438\end{array}$ & $\begin{array}{l}1700 \\
1543 \\
1700\end{array}$ & $\begin{array}{r}65 \\
71 \\
194\end{array}$ & $\begin{array}{l}10 \\
11 \\
30\end{array}$ & $\begin{array}{l}6.2 \\
3.6 \\
9.3\end{array}$ & $\begin{array}{r}8 \\
7 \\
28\end{array}$ & $\begin{array}{l}65.05 \\
68.35 \\
58.62\end{array}$ & $\begin{array}{l}.42169 \\
.36878 \\
.52059\end{array}$ \\
\hline
\end{tabular}

\begin{tabular}{|c|c|c|c|c|c|c|c|c|c|c|c|c|}
\hline $\begin{array}{l}36 \\
\mathrm{NBA}-\mathrm{BO} \\
\mathrm{GBR}-\mathrm{BO}\end{array}$ & $16 \mathrm{Mov}$ & 2 & 1603 & 1533 & 1550 & 1650 & 26 & 4 & 2.2 & 3 & $\begin{array}{l}55.39 \\
78.65\end{array}$ & $\begin{array}{l}.56793 \\
.19674\end{array}$ \\
\hline $\begin{array}{l}N B A-C O \\
N B A-M A \\
N B A-E A\end{array}$ & & & & $\begin{array}{l}1537 \\
1538 \\
1536\end{array}$ & $\begin{array}{l}1550 \\
1552 \\
1549\end{array}$ & $\begin{array}{l}1632 \\
1640 \\
1638\end{array}$ & $\begin{array}{r}19 \\
117 \\
58\end{array}$ & $\begin{array}{r}3 \\
18 \\
9\end{array}$ & $\begin{array}{l}0.9 \\
5.0 \\
2.3\end{array}$ & $\begin{array}{r}3 \\
22 \\
8\end{array}$ & $\begin{array}{l}63.47 \\
63.35 \\
60.21\end{array}$ & $\begin{array}{r}.44663 \\
.44849 \\
.49670\end{array}$ \\
\hline $\begin{array}{l}37 \\
\text { GBR-BO } \\
\text { NBA-BO } \\
\text { NBA-MA }\end{array}$ & $2 \mathrm{DeC}$ & 1 & 1920 & $\begin{array}{l}1921 \\
1923 \\
1924\end{array}$ & $\begin{array}{l}1926 \\
1932 \\
1930\end{array}$ & $\begin{array}{l}1933 \\
1950 \\
1956\end{array}$ & $\begin{array}{l}11 \\
19 \\
52\end{array}$ & $\begin{array}{l}2 \\
3 \\
8\end{array}$ & $\begin{array}{l}0.6 \\
1.8 \\
2.5\end{array}$ & $\begin{array}{r}4 \\
1 \\
26\end{array}$ & $\begin{array}{l}75.92 \\
52.85 \\
45.49\end{array}$ & $\begin{array}{l}.24320 \\
.60377 \\
.70094\end{array}$ \\
\hline
\end{tabular}




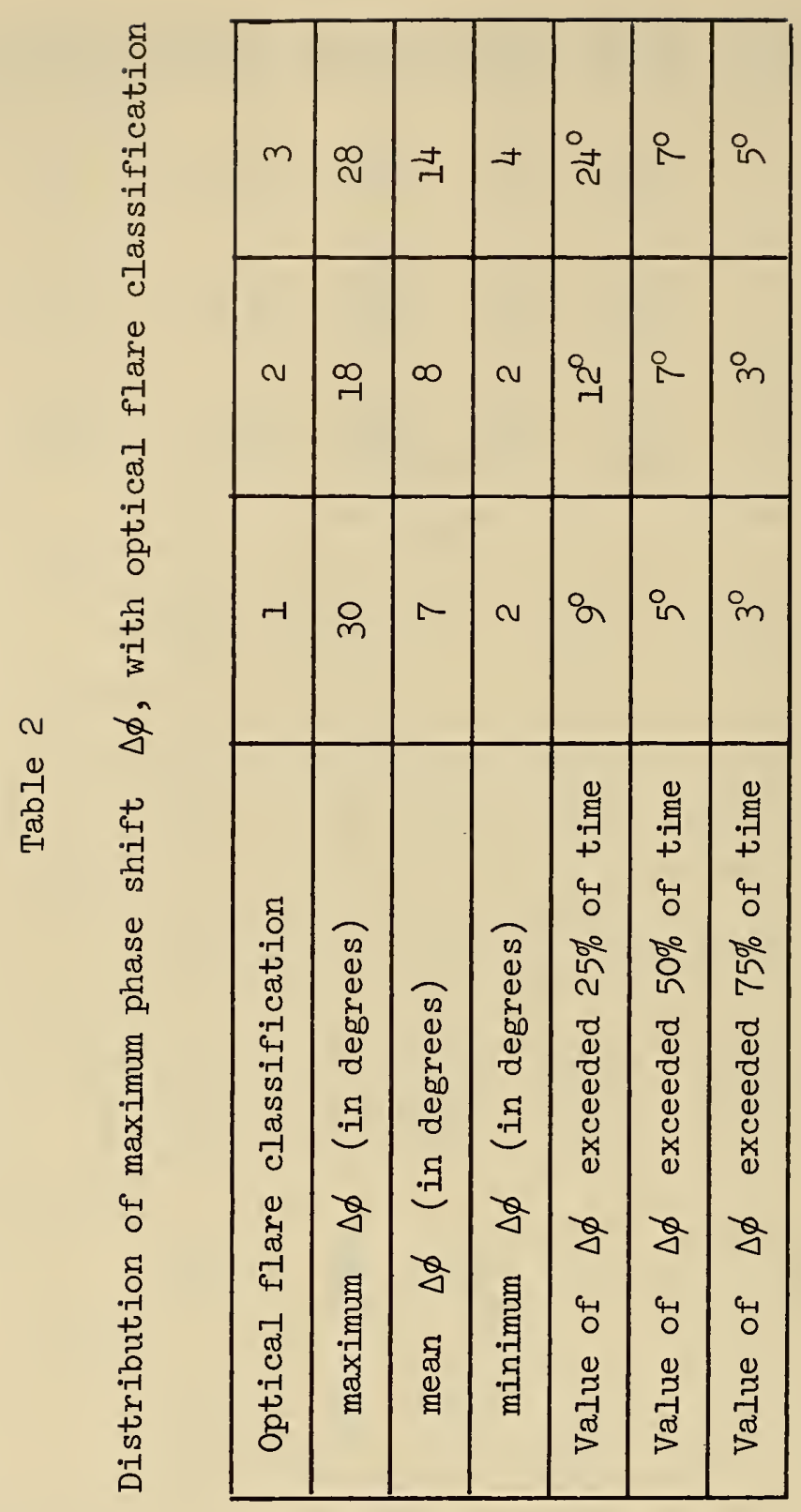




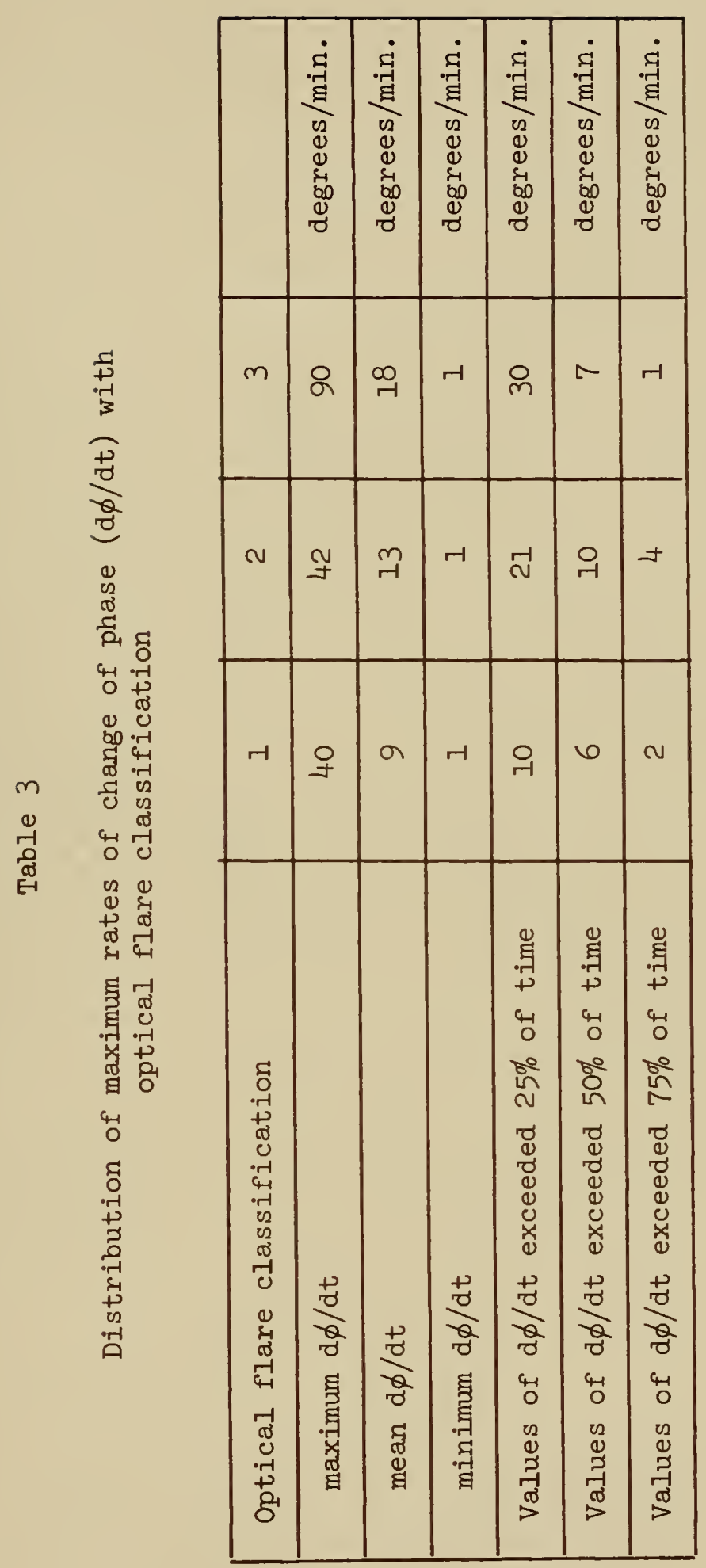




(1) 


